\title{
A CALCULUS FOR A CLASS OF FINITELY DEGENERATE PSEUDODIFFERENTIAL OPERATORS
}

\author{
INGO WITT \\ Institute of Mathematics, University of Potsdam \\ P.O. Box 6015 53, D-14415 Potsdam, Germany \\ E-mail:ingo@math.uni-potsdam.de
}

\begin{abstract}
For a class of degenerate pseudodifferential operators, local parametrices are constructed. This is done in the framework of a pseudodifferential calculus upon adding conditions of trace and potential type, respectively, along the boundary on which the operators degenerate.
\end{abstract}

1. Introduction. Given $l_{*} \in \mathbb{N}, l_{*} \geq 1$, the aim of this paper is to construct, locally near $t=0$, parametrices to differential operators of the form

$$
A=\sum_{j+|\alpha| \leq m} a_{j \alpha}(t, x) t^{\left(j+\left(l_{*}+1\right)|\alpha|-m\right)^{+}} D_{t}^{j} D_{x}^{\alpha}, \quad(t, x) \in(0, T) \times \Omega,
$$

and to related pseudodifferential operators. In (1.1), $m \in \mathbb{N}, T>0$ (or $T=\infty$ ), $\Omega \subseteq \mathbb{R}^{n}$ is an open subset, $a_{j \alpha} \in C^{\infty}([0, T) \times \Omega)$ for $j+|\alpha| \leq m$, and $y^{+}:=\max \{y, 0\}$ for $y \in \mathbb{R}$.

1.1. Motivation. The operator $A$ degenerates in a typical manner as $t \rightarrow+0$. In a well-defined sense, this degeneracy is characterized by the function $\lambda(t)=t^{l_{*}}$, see Yagdjian [24] for such an approach (and also Dreher [4] in this volume). Connected with that, ellipticity of $A$ manifests in the invertibility on the level of two principal symbols: The usual interior principal symbol,

$$
\sigma_{\psi}^{m}(A)(t, x, \tau, \xi):=\sum_{j+|\alpha|=m} a_{j \alpha}(t, x) t^{l_{*}|\alpha|} \tau^{j} \xi^{\alpha},
$$

and the boundary principal symbol,

$$
\sigma_{b}^{m ; \lambda}(A)(x, \xi):=\sum_{\substack{j+|\alpha| \leq m, j+\left(l_{*}+1\right)|\alpha| \geq m}} a_{j \alpha}(0, x) t^{j+\left(l_{*}+1\right)|\alpha|-m} D_{t}^{j} \xi^{\alpha} .
$$

2000 Mathematics Subject Classification: Primary 35J70, 35S15; Secondary 35J40, 35H10.

Key words and phrases: Pseudodifferential calculus, finitely degenerate pseudodifferential operators, boundary principal symbol, principal symbol map, ellipticity, local parametrix construction.

The paper is in final form and no version of it will be published elsewhere. 
Note that $\sigma_{b}^{m ; \lambda}(A)(x, \xi)$ is a family of ordinary differential operators on the half-line $\mathbb{R}_{+}$ depending on $(x, \xi) \in \Omega \times\left(\mathbb{R}^{n} \backslash 0\right)$. Here and in the following, superscript $\lambda$ is used for designating objects whose definitions depend on the considered type of degeneracy.

REMARK 1.1. Under the assumption that $\tilde{\sigma}_{\psi}^{m ; \lambda}(A)(t, x, \tau, \tilde{\xi}) \neq 0$ for all $(t, x, \tau, \tilde{\xi}) \in$ $[0, T) \times \Omega \times\left(\mathbb{R}^{1+n} \backslash 0\right)$, where the symbol $\tilde{\sigma}_{\psi}^{m ; \lambda}(A)$ is related to $\sigma_{\psi}^{m}(A)$ via

$$
\sigma_{\psi}^{m}(A)(t, x, \tau, \xi)=\tilde{\sigma}_{\psi}^{m ; \lambda}(A)\left(t, x, \tau, t^{l_{*}} \xi\right)
$$

we already have that, for each $(x, \xi) \in \Omega \times\left(\mathbb{R}^{n} \backslash 0\right), \sigma_{b}^{m ; \lambda}(A)(x, \xi)$ considered as acting in $\mathcal{S}\left(\overline{\mathbb{R}}_{+}\right)$is a Fredholm operator. In fact, its index equals the number of zeros in $\tau$ with $\Im \tau>0$ of the polynomial equation $\tilde{\sigma}_{\psi}^{m ; \lambda}(A)(0, x, \tau, \tilde{\xi})=0$, see Levendorskii [15, Theorem 2.5.1].

To achieve invertibility of the operators $\sigma_{b}^{m ; \lambda}(A)(x, \xi)$, we have, in general, to add boundary conditions at $t=0$.

Thus, the program to carry out is as follows:

- Construct a pseudodifferential calculus containing differential operators $A$ of the form (1.1) as well as the parametrices to the elliptic elements within the calculus.

- Ellipticity is determined by invertibility on the level of two principal symbols, as indicated by (1.2), (1.3).

- Add trace and potential operators like in Boutet de Monvel's calculus for pseudodifferential boundary value problems, for the latter, see Boutet de Monvel [2], RempelSchulze [18], Schulze [20].

Then the concrete form of symbol estimates, as given in (4.1), are predicted by two further observations: The first one concerns weakly hyperbolic operators $A$ of the form (1.1), where $t$ is now time. The Cauchy problem for $A$ with data posed at $t=0$ is $C^{\infty}$-well-posed, see, e.g., Ivrii-Petkov [12]. In Dreher-Witt [5], see also Dreher [4], Sobolev-type spaces $H_{\text {loc }}^{s, \delta ; \lambda}([0, T) \times \Omega)$ (here subscript "loc" refers to the behaviour as $t \rightarrow T$ or as $x \rightarrow \partial \Omega$, whereas, in $H_{\text {loc }}^{s}((0, T) \times \Omega)$ used below, "loc" goes with the usual meaning) have been introduced in which energetic estimates for the operator $A$ are possible, in this way reflecting $C^{\infty}$-well-posedness. Here, $s$ stands for the usual Sobolev regularity, while $\delta \in \mathbb{R}$ is an additional parameter connected with the loss of regularity observed when going over from Cauchy data to the solution. In particular, $H_{\mathrm{loc}}^{s, \delta ; \lambda}([0, T) \times \Omega) \subset H_{\mathrm{loc}}^{s}((0, T) \times \Omega)$ with a special behaviour of functions in the first space as $t \rightarrow+0$. It turns out that the global version $H^{s, \delta ; \lambda}\left(\mathbb{R}_{+} \times \mathbb{R}^{n}\right)$ of these spaces can be obtained as

$$
H^{s, \delta ; \lambda}\left(\mathbb{R}_{+} \times \mathbb{R}^{n}\right)=\left.H\left(\Phi^{s} \phi^{s+\delta}, g\right)\right|_{\mathbb{R}_{+} \times \mathbb{R}^{n}},
$$

where $H\left(\Phi^{s} \phi^{s+\delta}, g\right)$ is a function space on $\mathbb{R} \times \mathbb{R}^{n}$ defined by a certain Hörmander metric $g$ (here not further specified) and $g$-admissible weight functions $\Phi, \phi$,

$$
\Phi(t, \tau, \xi)=1+|\tau|+|t|^{l_{*}}\langle\xi\rangle+|\xi|^{\beta_{*}}, \quad \phi(t, \xi)=\Phi(t, 0, \xi)^{-1},
$$

and $\beta_{*}=1 /\left(l_{*}+1\right)$. (For generalities on the Beals-Fefferman-Hörmander calculus, see Beals [1], Hörmander [10], Levendorskii [15].) 
REMARK 1.2. In terms of pseudodifferential operators, the space $H\left(\Phi^{s} \phi^{s+\delta}, g\right)$ for $s \geq 0, \delta \geq 0$ consists of all $u \in L^{2}\left(\mathbb{R} \times \mathbb{R}^{n}\right)$ for which

$$
\bar{\Phi}\left(t, D_{t}, D_{x}\right)^{s} \bar{\phi}\left(t, D_{x}\right)^{s+\delta} u \in L^{2}\left(\mathbb{R} \times \mathbb{R}^{n}\right) .
$$

where $\bar{\Phi}=\bar{\Phi}(t, \tau, \xi)$ is a smoothed version of $\Phi$, and $\bar{\phi}=\bar{\Phi}(t, 0, \xi)^{-1}$.

For the operator $A$ from (1.1),

$$
A: H_{\mathrm{loc}}^{s, \delta ; \lambda}([0, T) \times \Omega) \rightarrow H_{\mathrm{loc}}^{s-m, \delta+m ; \lambda}([0, T) \times \Omega) .
$$

We infer that $\Phi(t, \tau, \xi)^{m}$ is the basic weight function to choose for symbols $a(t, x, \tau, \xi)$ for operators $A=\mathrm{op}^{+}(a)$ of order $m$ in the calculus (for the notation op ${ }^{+}(a)$, see (2.3)).

The second observation equally comes from weakly hyperbolic theory and concerns the estimation of derivatives of $a(t, x, \tau, \xi)$. It is known, see, e.g., Yagdjian [24], that the microlocal analysis of the operator $A$ from (1.1) is different in the two zones in $(t, \xi)$-space given by $t^{l_{*}+1}\langle\xi\rangle \leq 1$ and $t^{l_{*}+1}\langle\xi\rangle \geq 1$, respectively: When $t^{l_{*}+1}\langle\xi\rangle \leq 1$, we can (essentially) take the estimates which are predicted by the weight functions $\Phi, \phi$, e.g., with each $t$-derivative we gain $\phi$ once (actually, one loses the weight $1+t^{l_{*}}\langle\xi\rangle+|\xi|^{\beta_{*}}$ ). When $t^{l_{*}+1}\langle\xi\rangle \geq 1$, however, as shown in Yagdjian [24, Chapter 3], this estimate has to be improved to the gain of $t^{-1}$.

REMARK 1.3. For the reader's convenience, to judge these assertions let us state the different behaviour of weight functions in these two zones: We have $t^{l_{*}}\langle\xi\rangle \geq\langle\xi\rangle^{\beta_{*}} \geq t^{-1}$ when $t^{l_{*}+1}\langle\xi\rangle \geq 1$, while $t^{l_{*}}\langle\xi\rangle \leq\langle\xi\rangle^{\beta_{*}} \leq t^{-1}$ when $t^{l_{*}+1}\langle\xi\rangle \leq 1$. Hence,

$$
\Phi(t, \tau, \xi) \sim\left\{\begin{array}{ll}
|\tau|+t^{l_{*}}\langle\xi\rangle & \text { if } t^{l_{*}+1}\langle\xi\rangle \geq 1 \\
|\tau|+\langle\xi\rangle^{\beta_{*}} & \text { if } t^{l_{*}+1}\langle\xi\rangle \leq 1
\end{array}, \quad\langle\xi\rangle^{-\beta_{*}}+t \sim\left\{\begin{array}{ll}
t & \text { if } t^{l_{*}+1}\langle\xi\rangle \geq 1 \\
\langle\xi\rangle^{-\beta_{*}} & \text { if } t^{l_{*}+1}\langle\xi\rangle \leq 1
\end{array} .\right.\right.
$$

Here, $\langle\xi\rangle^{-\beta_{*}}+t$ is the weight function used later to replace $\phi(t, \xi)$.

All together, the estimates (4.1) follow. Having estimates on the interior symbols, there still remain two points open: One concerns an appropriate formulation of the transmission property in the present situation (necessary, in order to keep $C^{\infty}$-smoothness up to $t=0$ in the mapping properties of pseudodifferential operators involved), the other one concerns the appearance of singular Green operators. Fortunately, we can provide a natural answer in both cases. Then, having resolved all the mentioned difficulties, the pseudodifferential calculus to develop is organized in a way as it has been done for Boutet de Monvel's calculus in Schulze [20, Chapter 4]. (This corresponds to the case in which, formally, $l_{*}=0$.) Therefore, we follow the exposition in this reference as close as possible, where possible.

1.2. Outline of the argument. The construction of the pseudodifferential calculus is oriented towards Boutet de Monvel's calculus for treating pseudodifferential boundary value problems, see Boutet de Monvel [2], Rempel-Schulze [18], Schulze [20]. In particular, operators have an order $m$ (for the sake of simplicity, $m$ is always assumed to be an integer) and a type $d \in \mathbb{N}$.

- Operators in the class $B^{m, d ; \lambda}\left((0, T) \times \Omega ; N_{-}, N_{+}\right)$(although all occurring operators are classical ones, this not specially mentioned in the notation in order not to overload 
it) are block matrices of the form

$$
\mathfrak{A}=\left(\begin{array}{cc}
\mathrm{op}^{+}(a)+G & K \\
S & Q
\end{array}\right): \begin{gathered}
C_{\mathrm{comp}}^{\infty}([0, T) \times \Omega) \\
C_{\mathrm{comp}}^{\infty}\left(\Omega ; \mathbb{C}^{N_{-}}\right)
\end{gathered} \rightarrow \begin{gathered}
C^{\infty}([0, T) \times \Omega) \\
C^{\infty}\left(\Omega ; \mathbb{C}^{N_{+}}\right)
\end{gathered}
$$

where $\mathrm{op}^{+}(a)$ takes the part of the operator $A$ from (1.1), $S$ is a trace operator of type $d, K$ is a potential operator, and $Q$ is a pseudodifferential operator acting along the boundary. For the special operator convention op ${ }^{+}(a)$, see (2.3). In particular, the symbol $a=a(t, x, \tau, \xi)$ has to satisfy the $\lambda$-transmission property with respect to $t=0$, as discussed in Section 4.2. Furthermore, $G$ is a so-called singular Green operator that is smoothing for $t>0$, but of the same type $d$, and arises in compositions.

- Operators act in the spaces $H_{\text {comp }}^{s, \delta ; \lambda}([0, T) \times \Omega)$ and $H_{\text {loc }}^{s, \delta ; \lambda}([0, T) \times \Omega)$, respectively. After performing suitable order reductions on the boundary (which we always assume to be done),

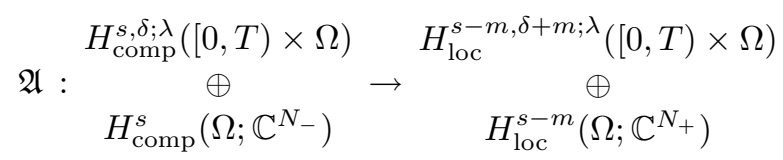

for $s>d-1 / 2$. In the case that $\mathfrak{A}$ is properly supported, one can even replace either "comp" by "loc" in the source space column or "loc" by "comp" in the target space column.

- Ellipticity is determined through invertibility on the symbolic level. More precisely, we have a pair $\left(\sigma_{\psi}^{m}(\mathfrak{A}), \sigma_{b}^{m ; \lambda}(\mathfrak{A})\right)$ of principal symbols, where $\sigma_{\psi}^{m}(\mathfrak{A})=\sigma_{\psi}^{m}\left(\mathrm{op}^{+}(a)\right)$ is the usual interior principal symbol and $\sigma_{b}^{m ; \lambda}(\mathfrak{A})$ is the boundary principal symbol that is operator-valued:

$$
\sigma_{b}^{m ; \lambda}(\mathfrak{A})(x, \xi): \quad \bigoplus^{H^{s, \delta ; \lambda}\left(\mathbb{R}_{+}\right)} \rightarrow \begin{gathered}
H^{s-m, \delta+m ; \lambda}\left(\mathbb{R}_{+}\right) \\
\mathbb{C}^{N_{-}}
\end{gathered} \quad \mathbb{C}^{N_{+}}
$$

for $(x, \xi) \in \Omega \times\left(\mathbb{R}^{n} \backslash 0\right)$. (Note that, as compared to the situation occurring in (1.8), the spaces $H^{s, \delta ; \lambda}\left(\mathbb{R}_{+}\right)$are now "global" ones.) In fact, $\sigma_{b}^{m ; \lambda}(\mathfrak{A})(x, \xi)$ belongs to the corresponding (global) pseudodifferential calculus on the half-line $\mathbb{R}_{+}$, i.e., $\sigma_{b}^{m ; \lambda}(\mathfrak{A})(x, \xi) \in$ $B^{m, d ; \lambda}\left(\mathbb{R}_{+} ; N_{-}, N_{+}\right)$for all $(x, \xi) \in \Omega \times\left(\mathbb{R}^{n} \backslash 0\right)$, and, in addition, we have "twisted" homogeneity, i.e.

$$
\sigma_{b}^{m ; \lambda}(\mathfrak{A})(x, \nu \xi)=\nu^{m}\left(\begin{array}{cc}
\kappa_{\nu}^{(\delta+m)} & 0 \\
0 & 1_{\mathbb{C}^{N_{+}}}
\end{array}\right) \sigma_{b}^{m ; \lambda}(\mathfrak{A})(x, \xi)\left(\begin{array}{cc}
\kappa_{\nu}^{(\delta)} & 0 \\
0 & 1_{\mathbb{C}^{N_{-}}}
\end{array}\right)^{-1}
$$

for $\nu>0$, where

$$
\kappa_{\nu}^{(\delta)} v(t)=\nu^{\beta_{*} / 2-\beta_{*} \delta l_{*}} v\left(\nu^{\beta_{*}} t\right)
$$

for functions $v=v(t)$ on $\mathbb{R}_{+} ; \delta \in \mathbb{R}$ is arbitrary.

REMARK 1.4. It is seen that $\left\{\kappa_{\nu}^{(\delta)}\right\}_{\nu>0}$ constitutes a strongly continuous group of bounded operators acting on $H^{s, \delta ; \lambda}\left(\mathbb{R}_{+}\right)$; especially, $\kappa_{1}^{(\delta)}=1, \kappa_{\nu}^{(\delta)} \kappa_{\nu^{\prime}}^{(\delta)}=\kappa_{\nu \nu^{\prime}}^{(\delta)}$ for $\nu, \nu^{\prime}>0$. 
In a more general context, this observation serves as starting point for Schulze's abstract edge approach, see Schulze [20, Section 1.3, Chapters 3-4].

In our case, the "abstract edge" is identified with the boundary.

1.3. Main results. Next, let us state the main results of this paper.

Theorem 1.5. For $\mathfrak{A} \in B^{m, d ; \lambda}\left((0, T) \times \Omega ; \tilde{N}, N_{+}\right), \mathfrak{B} \in B^{m^{\prime}, d^{\prime} ; \lambda}\left((0, T) \times \Omega ; N_{-}, \tilde{N}\right)$, with at least one of these operators properly supported,

$$
\mathfrak{A} \mathfrak{B} \in B^{m+m^{\prime}, d^{\prime \prime} ; \lambda}\left((0, T) \times \Omega ; N_{-}, N_{+}\right),
$$

where $d^{\prime \prime}=\max \left\{d+m^{\prime}, d^{\prime}\right\}$. Furthermore,

$$
\sigma_{\psi}^{m+m^{\prime}}(\mathfrak{A} \mathfrak{B})=\sigma_{\psi}^{m}(\mathfrak{A}) \sigma_{\psi}^{m^{\prime}}(\mathfrak{B}), \quad \sigma_{b}^{m+m^{\prime} ; \lambda}(\mathfrak{A} \mathfrak{B})=\sigma_{b}^{m ; \lambda}(\mathfrak{A}) \sigma_{b}^{m^{\prime} ; \lambda}(\mathfrak{B}) .
$$

Now let

$$
\Sigma B^{m, d ; \lambda}\left((0, T) \times \Omega ; N_{-}, N_{+}\right):=\left\{\left(\sigma_{\psi}^{m}(\mathfrak{A}), \sigma_{b}^{m ; \lambda}(\mathfrak{A})\right) ; \mathfrak{A} \in B^{m, d ; \lambda}\left((0, T) \times \Omega ; N_{-}, N_{+}\right)\right\}
$$

denote the principal symbol space of $B^{m, d ; \lambda}\left((0, T) \times \Omega ; N_{-}, N_{+}\right)$. (An intrinsic description is provided in Definition 5.5.)

THEOREM 1.6. The following short sequence is split exact:

$$
\begin{aligned}
0 \longrightarrow B^{m-1, d ; \lambda}\left((0, T) \times \Omega ; N_{-}, N_{+}\right) & \longrightarrow B^{m, d ; \lambda}\left((0, T) \times \Omega ; N_{-}, N_{+}\right) \\
& \stackrel{\sigma^{m ; \lambda}}{\longrightarrow} \Sigma B^{m, d ; \lambda}\left((0, T) \times \Omega ; N_{-}, N_{+}\right) \longrightarrow 0,
\end{aligned}
$$

where $\sigma^{m ; \lambda}=\left(\sigma_{\psi}^{m}, \sigma_{b}^{m ; \lambda}\right)$ is the principal symbol map.

REMARK 1.7. Operators $\mathfrak{A} \in B^{m-1, d ; \lambda}\left((0, T) \times \Omega ; N_{-}, N_{+}\right)$are (locally) compact when considered as acting in (1.8). This underscores the importance of the previous theorem.

Theorems 1.5 and 1.6 together yield in a standard manner the following result:

ThEOREM 1.8. Let $\mathfrak{A} \in B^{m, d ; \lambda}\left((0, T) \times \Omega ; N_{-}, N_{+}\right)$be elliptic in the sense that both principal symbols $\sigma_{\psi}^{m}(\mathfrak{A})$ and $\sigma_{b}^{m ; \lambda}(\mathfrak{A})$ are invertible, the first one when $t>0$, the second one when $t=0$. Then there is a parametrix $\mathfrak{B}$ to $\mathfrak{A}$, i.e., a properly supported operator $\mathfrak{B} \in B^{-m,(d-m)^{+} ; \lambda}\left((0, T) \times \Omega ; N_{+}, N_{-}\right)$such that

$$
\begin{aligned}
& \mathfrak{B A}-1 \in B^{-\infty, \max \{m, d\} ; \lambda}\left((0, T) \times \Omega ; N_{-}, N_{-}\right), \\
& \mathfrak{A} \mathfrak{B}-1 \in B^{-\infty,(d-m)^{+} ; \lambda}\left((0, T) \times \Omega ; N_{+}, N_{+}\right) .
\end{aligned}
$$

Operators with the kind of degeneracy as described by (1.1) were considered by many authors under various aspects, e.g., local solvability, hypo-ellipticity, parametrix constructions. Let us mention at least some related work and refer to the references therein: Boutet de Monvel [3], Grushin [6], [7], Hanges [8], Joshi [13], Levendorskii [15], MascarelloRodino [16], Sjöstrand [21], Vishik-Grushin [23], Yagdjian [24], and many others.

The paper is organized as follows: In Section 2, we recall some notation and basic properties of the function spaces used. In Section 3, the calculus on the half-line, in which the boundary symbols take their values, is developed. Section 4 is then devoted to the boundary symbolic calculus. Eventually, in Section 5, the (local) complete pseudodifferential calculus follows. This includes indications of the proofs of the main theorems. 
Acknowledgements. I am grateful to Prof. M. Reissig (Freiberg University of Mining and Technology) for several comments on the manuscript, in particular, concerning Examples 3.10 and 5.8.

2. Preliminaries. We shall exploit standard notation as found, for example, in Hörmander [11], Kumano-go [14], Taylor [22]. Moreover, we shall make use of the following notation and function spaces:

2.1. Notation. The symbol $\chi=\chi(t)$ is always chosen to denote an excision function, i.e., $\chi \in C^{\infty}(\mathbb{R})$ is such that $\chi(t)=0$ for $|t| \leq 1 / 2$ and $\chi(t)=1$ for $|t| \geq 1$.

The symbol $S^{m}(\ldots)$ serves to denote spaces of pseudodifferential symbols of order $m \in \mathbb{R}$ and type 1,0 , while spaces of classical pseudodifferential symbols of order $m$, i.e., symbols possessing asymptotic expansions into homogeneous components decreasing by one each step, are denoted by $S_{\mathrm{cl}}^{m}(\ldots)$. Accordingly, spaces of pseudodifferential symbols of order $m$ and type 1,0 possessing the transmission property are denoted by $S_{\mathrm{tr}}^{m}(\ldots)$. Finally, we write $S_{\mathrm{tr}, \mathrm{cl}}^{m}(\ldots):=S_{\mathrm{tr}}^{m}(\ldots) \cap S_{\mathrm{cl}}^{m}(\ldots)$.

We shall also encounter spaces of classical pseudodifferential symbols of order $m^{\prime} \in \mathbb{R}$, where the homogeneity of components goes down by $\beta_{*}$ each step (recall that $\beta_{*}=\frac{1}{l_{*}+1}$ ). These spaces are denoted by $S_{\mathrm{cl}, \beta_{*}}^{m^{\prime}}(\ldots)$. In such a case, we always have $m^{\prime} \in \beta_{*} \mathbb{Z}$.

Let us recall what is meant by saying that a pseudodifferential symbol has the transmission property (with respect to $t=0$ ), see Rempel-Schulze [18]: For $m \in \mathbb{Z}$, let $H_{m}$ be the space of all functions $h \in C^{\infty}(\mathbb{R})$ which possess an asymptotic expansion

$$
h(\tau) \sim \sum_{k=-m}^{\infty} h_{k} \tau^{-k-1} \quad \text { as }|\tau| \rightarrow \infty
$$

for certain $h_{k} \in \mathbb{C}$ which can be formally differentiated any number of times. Then $a \in S^{m}\left([0, T) \times \Omega \times \mathbb{R}^{1+n}\right)$ has the transmission property if

$$
\partial_{t}^{k} \partial_{x}^{\beta} a(0, x,\langle\xi\rangle \tau, \xi) \in S^{m}\left(\Omega \times \mathbb{R}^{n} ; H_{m+1}\right)
$$

for all $k \in \mathbb{N}, \beta \in \mathbb{N}^{n}$. If $a \in S_{\mathrm{cl}}^{m}\left([0, T) \times \Omega \times \mathbb{R}^{1+n}\right), m \in \mathbb{Z}$, and

$$
a \sim \sum_{j=0}^{\infty} \chi(|\tau, \xi|) a_{j}(t, x, \tau, \xi),
$$

where $a_{j} \in S^{(m-j)}\left([0, T) \times \Omega \times\left(\mathbb{R}^{1+n} \backslash 0\right)\right)$ for $j=0,1,2 \ldots$, is the asymptotic expansion of $a$ into homogeneous components, then (2.2) holds if and only if

$$
\partial_{t}^{k} \partial_{\xi}^{\alpha} a_{j}(0, x,-1, \xi)=(-1)^{m-j-|\alpha|} \partial_{t}^{k} \partial_{\xi}^{\alpha} a_{j}(0, x, 1, \xi)
$$

for all $j, k \in \mathbb{N}, \alpha \in \mathbb{N}^{n}$.

If $a(t, \tau)$ is a pseudodifferential symbol on $\overline{\mathbb{R}}_{+} \times \mathbb{R}$ (in our case, always with the transmission property with respect to $t=0)$, then $\mathrm{op}^{+}(a)$ is the pseudodifferential operator defined by

$$
\mathrm{op}^{+}(a)=r^{+} \mathrm{op}(\tilde{a}) e^{+},
$$

where $e^{+}$is extension by zero from $\mathbb{R}_{+}$to $\mathbb{R}, r^{+}$restriction from $\mathbb{R}$ to $\mathbb{R}_{+}, \tilde{a}$ is any extension of $a$ to a pseudodifferential symbol on $\mathbb{R} \times \mathbb{R}$, and op $(\tilde{a})$ refers to the standard quan- 
tization, i.e., $\operatorname{op}(\tilde{a}) u(t)=(2 \pi)^{-1} \int_{-\infty}^{\infty} e^{i t \tau} \tilde{a}(t, \tau) \hat{u}(\tau) d \tau$, where $\hat{u}(\tau)=\int_{-\infty}^{\infty} e^{-i t \tau} u(t) d t$ is the Fourier transform of $u$. This definition is independent of the choice of the extension of $a$. Indeed, we have

$$
\mathrm{op}^{+}(a) u(t)=(2 \pi)^{-1} \int_{-\infty}^{\infty} e^{i t \tau} a(t, \tau) \hat{u}(\tau) d \tau, \quad t>0
$$

where now $\hat{u}(\tau)=\int_{0}^{\infty} e^{-i t \tau} u(t) d t$. By pseudo-locality, $\operatorname{op}^{+}(a)$ is a pseudodifferential operator on $\mathbb{R}_{+}$of the corresponding order.

The same convention applies for $\mathrm{op}^{+}(a)$ (again with respect to $\left.t=0\right)$ if $a(t, x, \tau, \xi)$ is a pseudodifferential symbol on $[0, T) \times \Omega \times \mathbb{R}^{1+n}$, i.e., we have op ${ }^{+}(a)=\mathrm{op}_{x}\left(\mathrm{op}_{t}^{+}(a)\right)$, where the variables with respect to which the action is performed are explicitly indicated.

2.2. Function spaces. The function spaces $H^{s, \delta ; \lambda}\left(\mathbb{R}_{+} \times \mathbb{R}^{n}\right)$ for $s \geq 0, \delta \in \mathbb{R}$ have been introduced in Dreher-Witt [5] by combining ideas from weakly hyperbolic theory and Schulze's abstract edge approach, for the latter, see Schulze [20].

Let us recall the basic definitions and some relevant properties, see also Dreher [4]: On the half-line $\mathbb{R}_{+}$, the space $H^{s, \delta ; \lambda}\left(\mathbb{R}_{+}\right)$for $s, \delta \in \mathbb{R}$ is explained as the space of all $u \in H_{\text {loc }}^{s}\left(\mathbb{R}_{+}\right)$such that, for any excision function $\chi,(1-\chi(t)) u \in H^{s}\left(\mathbb{R}_{+}\right)$and

$$
\left(t^{-l_{*}} \partial_{t}\right)^{j} \chi(t) u \in t^{\delta l_{*}} L^{2}\left(\mathbb{R}_{+}\right), \quad j=0,1, \ldots, s,
$$

the latter provided that $s \in \mathbb{N}$. The case of an arbitrary $s \in \mathbb{R}$ is treated by interpolation and duality.

The space $H^{s, \delta ; \lambda}\left(\mathbb{R}_{+} \times \mathbb{R}^{n}\right)$ is then introduced as the abstract edge Sobolev space of smoothness $s$ with respect to the space $H^{s, \delta ; \lambda}\left(\mathbb{R}_{+}\right)$and the strongly continuous group action $\left\{\kappa_{\mu}^{(\delta)}\right\}_{\mu>0}$ on it, see (1.9). This means that $H^{s, \delta ; \lambda}\left(\mathbb{R}_{+} \times \mathbb{R}^{n}\right)$ is the space of all tempered distributions $u \in \mathcal{S}^{\prime}\left(\mathbb{R}^{n} ; H^{s, \delta ; \lambda}\left(\mathbb{R}_{+}\right)\right.$) (with values in $H^{s, \delta ; \lambda}\left(\mathbb{R}_{+}\right)$) such that $\hat{u} \in L_{\text {loc }}^{2}\left(\mathbb{R}^{n} ; H^{s, \delta ; \lambda}\left(\mathbb{R}_{+}\right)\right)$, where $\hat{u}(t, \xi)=\int_{\mathbb{R}^{n}} e^{-i x \xi} u(x) d x$ is the partial Fourier transform of $u$ with respect to $x$, and the norm

$$
\|u\|_{H^{s, \delta ; \lambda}\left(\mathbb{R}_{+} \times \mathbb{R}^{n}\right)}:=\left\{\int\langle\xi\rangle^{2 s}\left\|\kappa_{\langle\xi\rangle-1}^{(\delta)} \hat{u}(\cdot, \xi)\right\|_{H^{s, \delta ; \lambda}\left(\mathbb{R}_{+}\right)}^{2} d \xi\right\}^{1 / 2}
$$

is finite. The equivalence with the description provided in (1.4), (1.5) can be easily obtained by a direct computation.

We list properties of the spaces $H^{s, \delta ; \lambda}\left(\mathbb{R}_{+} \times \mathbb{R}^{n}\right)$. For most of the proofs, we refer to Dreher-Witt [5]:

(a) $H^{0,0 ; \lambda}\left(\mathbb{R}_{+} \times \mathbb{R}^{n}\right)=L^{2}\left(\mathbb{R}_{+} \times \mathbb{R}^{n}\right), H^{-s,-\delta ; \lambda}\left(\mathbb{R}_{+} \times \mathbb{R}^{n}\right)$ is the dual to $H^{s, \delta ; \lambda}\left(\mathbb{R}_{+} \times \mathbb{R}^{n}\right)$ with respect to an extension of the $L^{2}$-scalar product.

(b) $\left\{H^{s, \delta ; \lambda}\left(\mathbb{R}_{+} \times \mathbb{R}^{n}\right) ; s, \delta \in \mathbb{R}\right\}$ forms an interpolation scale of Hilbert spaces with respect to the complex interpolation method, jointly in both parameters $s, \delta$.

(c) For any $a>0$,

$$
\left.H^{s, \delta ; \lambda}\left(\mathbb{R}_{+} \times \mathbb{R}^{n}\right)\right|_{(a, \infty) \times \mathbb{R}^{n}}=\left\{t^{l_{*} / 2+\delta l_{*}} v\left(t^{l_{*}+1}, x\right) ; v \in H^{s}\left((a, \infty) \times \mathbb{R}^{n}\right)\right\},
$$

in particular, $H^{s, \delta ; \lambda}\left(\mathbb{R}_{+} \times \mathbb{R}^{n}\right) \subset H_{\text {loc }}^{s}\left(\mathbb{R}_{+} \times \mathbb{R}^{n}\right)$. 
(d) The space $\mathcal{S}\left(\overline{\mathbb{R}}_{+} \times \mathbb{R}^{n}\right)$ is dense in $H^{s, \delta ; \lambda}\left(\mathbb{R}_{+} \times \mathbb{R}^{n}\right)$, moreover,

$$
\bigcap_{s, \delta \in \mathbb{R}} H^{s, \delta ; \lambda}\left(\mathbb{R}_{+} \times \mathbb{R}^{n}\right)=\mathcal{S}\left(\overline{\mathbb{R}}_{+} \times \mathbb{R}^{n}\right) .
$$

(e) For any $s>1 / 2$, the trace operator

$$
\begin{aligned}
H^{s, \delta ; \lambda}\left(\mathbb{R}_{+} \times \mathbb{R}^{n}\right) & \rightarrow \bigoplus_{j=0}^{j_{0}} H^{s-\beta_{*} j+\beta_{*} \delta l_{*}-\beta_{*} / 2}\left(\mathbb{R}^{n}\right), \\
u & \mapsto\left(\gamma_{0} u, \gamma_{1} u, \ldots, \gamma_{j_{0}} u\right),
\end{aligned}
$$

where $\gamma_{j} u:=\left.\partial_{t}^{j} u\right|_{t=0}, j=0,1, \ldots, j_{0}$, and $j_{0}$ is the largest integer less than $s-1 / 2$, is surjective.

(f) For any $a>0,\left\{u \in H^{s, \delta ; \lambda}\left(\mathbb{R}_{+} \times \mathbb{R}^{n}\right)\right.$; $\left.\operatorname{supp} u \subseteq(0, a] \times \mathbb{R}^{n}\right\} \subseteq H^{s^{\prime}, \delta^{\prime} ; \lambda}\left(\mathbb{R}_{+} \times \mathbb{R}^{n}\right)$ if and only if $s \geq s^{\prime}, s+\beta_{*} \delta l_{*} \geq s^{\prime}+\beta_{*} \delta^{\prime} l_{*}$.

(g) For any $a>0, K \Subset \mathbb{R}^{n}$, the embedding $\left\{u \in H^{s, \delta ; \lambda}\left(\mathbb{R}_{+} \times \mathbb{R}^{n}\right)\right.$; $\operatorname{supp} u \subseteq(0, a] \times$ $K\} \subseteq H^{s^{\prime}, \delta^{\prime} ; \lambda}\left(\mathbb{R}_{+} \times \mathbb{R}^{n}\right)$ is compact if and only if $s>s^{\prime}, s+\beta_{*} \delta l_{*}>s^{\prime}+\beta_{*} \delta^{\prime} l_{*}$.

Eventually, the space $H_{\mathrm{loc}}^{s, \delta ; \lambda}([0, T) \times \Omega)$ is introduced as the space of all functions $u \in H_{\text {loc }}^{s}((0, T) \times \Omega)$ such that $\varphi u \in H^{s, \delta ; \lambda}\left(\mathbb{R}_{+} \times \mathbb{R}^{n}\right)$ for any $\varphi \in C_{\text {comp }}^{\infty}([0, T) \times \Omega)$. Furthermore, the space $H_{\text {comp }}^{s, \delta ; \lambda}([0, T) \times \Omega)$ is the space of all $u \in H_{\mathrm{loc}}^{s, \delta ; \lambda}([0, T) \times \Omega)$ satisfying $\operatorname{supp} u \Subset[0, T) \times \Omega$.

3. The calculus on the half-axis. The calculus on the half-line $\mathbb{R}_{+}$is, in essence, a calculus for a certain class of ordinary differential operators, and related pseudodifferential operators. The operators under consideration are the usual ones when $t \leq 1$, with smoothness preserved up to $t=0$. Therefore, the main contribution comes from the behaviour when $t \geq 1$. A related calculus has been studied in Mascarello-Rodino [16, Chapter 7]. In this reference, also a number of examples is thoroughly discussed.

The exposition here follows closely that in Schulze [20, Section 4.1]: There the case in which, formally, $l_{*}=0$ is treated; proofs carry over almost literally to the present situation.

Definition 3.1. For $m \in \mathbb{Z}$, the class $S^{m ; \lambda}\left(\overline{\mathbb{R}}_{+} \times \mathbb{R}\right)$ consists of all $a \in C^{\infty}\left(\overline{\mathbb{R}}_{+} \times \mathbb{R}\right)$ such that, for all $j, k \in \mathbb{N}$, there is a constant $C_{j k}>0$ such that

$$
\left|\partial_{t}^{k} \partial_{\tau}^{j} a(t, \tau)\right| \leq C_{j k}\left(1+|\tau|+t^{l_{*}}\right)^{m-j}(1+t)^{-k}
$$

for all $(t, \tau) \in \overline{\mathbb{R}}_{+} \times \mathbb{R}$. We further set

$$
S_{\mathrm{tr}, \mathrm{cl}}^{m ; \lambda}\left(\overline{\mathbb{R}}_{+} \times \mathbb{R}\right):=S^{m ; \lambda}\left(\overline{\mathbb{R}}_{+} \times \mathbb{R}\right) \cap S_{\mathrm{tr}, \mathrm{cl}}^{m}\left(\overline{\mathbb{R}}_{+} \times \mathbb{R}\right) .
$$

We have $S_{\mathrm{tr}, \mathrm{cl}}^{m-1 ; \lambda}\left(\overline{\mathbb{R}}_{+} \times \mathbb{R}\right) \subseteq S_{\mathrm{tr}, \mathrm{cl}}^{m ; \lambda}\left(\overline{\mathbb{R}}_{+} \times \mathbb{R}\right)$ and

$$
\bigcap_{m \in \mathbb{Z}} S_{\mathrm{tr}, \mathrm{cl}}^{m ; \lambda}\left(\overline{\mathbb{R}}_{+} \times \mathbb{R}\right)=\mathcal{S}\left(\overline{\mathbb{R}}_{+} \times \mathbb{R}\right) \text {. }
$$


Proposition 3.2. Let $a_{j} \in S_{\mathrm{tr}, \mathrm{cl}}^{m-j ; \lambda}\left(\overline{\mathbb{R}}_{+} \times \mathbb{R}\right)$ for $j=0,1,2, \ldots$ Then there is a symbol $a \in S_{\mathrm{tr}, \mathrm{cl}}^{m ; \lambda}\left(\overline{\mathbb{R}}_{+} \times \mathbb{R}\right)$ such that, for all $j \in \mathbb{N}$,

$$
a-\sum_{j<J} a_{j} \in S_{\mathrm{tr}, \mathrm{cl}}^{m-J}\left(\overline{\mathbb{R}}_{+} \times \mathbb{R}\right) .
$$

Proposition 3.3. For the symbol classes $S_{\mathrm{tr}, \mathrm{cl}}^{m ; \lambda}\left(\overline{\mathbb{R}}_{+} \times \mathbb{R}\right)$ for $m \in \mathbb{Z}$ the following rules hold:

(a) $\partial_{t}^{k} \partial_{\tau}^{j} a \in S_{\mathrm{tr}, \mathrm{cl}}^{m-j ; \lambda}\left(\overline{\mathbb{R}}_{+} \times \mathbb{R}\right)$ if $a \in S_{\mathrm{tr}, \mathrm{cl}}^{m ; \lambda}\left(\overline{\mathbb{R}}_{+} \times \mathbb{R}\right)$.

(b) $a b \in S_{\mathrm{tr}, \mathrm{cl}}^{m+m^{\prime} ; \lambda}\left(\overline{\mathbb{R}}_{+} \times \mathbb{R}\right)$ if $a \in S_{\mathrm{tr}, \mathrm{cl}}^{m ; \lambda}\left(\overline{\mathbb{R}}_{+} \times \mathbb{R}\right), b \in S_{\mathrm{tr}, \mathrm{cl}}^{m^{\prime} ; \lambda}\left(\overline{\mathbb{R}}_{+} \times \mathbb{R}\right)$.

(c) $a \circ b \in S_{\mathrm{tr}, \mathrm{cl}}^{m+m^{\prime} ; \lambda}\left(\overline{\mathbb{R}}_{+} \times \mathbb{R}\right)$ if $a \in S_{\mathrm{tr}, \mathrm{cl}}^{m ; j}\left(\overline{\mathbb{R}}_{+} \times \mathbb{R}\right), b \in S_{\mathrm{tr}, \mathrm{cl}}^{m^{\prime} ; \lambda}\left(\overline{\mathbb{R}}_{+} \times \mathbb{R}\right)$. Furthermore,

$$
(a \circ b)(t, \tau) \sim \sum_{j \in \mathbb{N}} \frac{1}{j !} \partial_{\tau}^{j} a(t, \tau) D_{t}^{j} b(t, \tau) .
$$

In Proposition $3.3(\mathrm{c})$, "о" is the Leibniz product, i.e.,

$$
(a \circ b)(t, \tau)=(2 \pi)^{-1} \iint e^{-i t^{\prime} \tau^{\prime}} a\left(t, \tau+\tau^{\prime}\right) b\left(t+t^{\prime}, \tau^{\prime}\right) d t^{\prime} d \tau^{\prime},
$$

understood as an oscillatory integral.

Definition 3.4. For $m \in \mathbb{Z}, d, N_{-}, N_{+} \in \mathbb{N}$, the class $B^{m, d ; \lambda}\left(\mathbb{R}_{+} ; N_{-}, N_{+}\right)$consists of all operator block matrices

$$
\mathfrak{A}=\left(\begin{array}{cc}
\mathrm{op}^{+}(a)+G & K \\
S & Q
\end{array}\right): \begin{array}{ccc}
\mathcal{S}\left(\overline{\mathbb{R}}_{+}\right) & \mathcal{S}\left(\overline{\mathbb{R}}_{+}\right) \\
\mathbb{C}^{N_{-}} & & \mathbb{C}^{N_{+}}
\end{array},
$$

where $a \in S_{\mathrm{tr}, \mathrm{cl}}^{m ; \lambda}\left(\overline{\mathbb{R}}_{+} \times \mathbb{R}\right)$,

$$
G u(t)=\sum_{k=0}^{d} \int_{0}^{\infty} g_{k}\left(t, t^{\prime}\right) \partial_{t^{\prime}}^{k} u\left(t^{\prime}\right) d t^{\prime},
$$

for some $g_{k} \in \mathcal{S}\left(\overline{\mathbb{R}}_{+} \times \overline{\mathbb{R}}_{+}\right), k=0,1, \ldots, d, S u=\left(S_{1} u, \ldots, S_{N_{+}} u\right)$,

$$
S_{j} u=\sum_{k=0}^{d} \int_{0}^{\infty} s_{j k}\left(t^{\prime}\right) \partial_{t^{\prime}}^{k} u\left(t^{\prime}\right) d t^{\prime}
$$

for some $s_{j k} \in \mathcal{S}\left(\overline{\mathbb{R}}_{+}\right), j=1, \ldots, N_{+}, k=0,1, \ldots, d$,

$$
K c=\sum_{l=1}^{N_{-}} c_{l} k_{l}(t), \quad c=\left(c_{1}, \ldots, c_{N_{-}}\right) \in \mathbb{C}^{N_{-}}
$$

for some $k_{l} \in \mathcal{S}\left(\overline{\mathbb{R}}_{+}\right), l=1, \ldots, N_{-}$, and $Q$ is a matrix of size $N_{+} \times N_{-}$.

In case $N_{-}=N_{+}=0$, we also write $B^{m, d ; \lambda}\left(\mathbb{R}_{+}\right):=B^{m, d ; \lambda}\left(\mathbb{R}_{+} ; 0,0\right)$. 
REMARK 3.5 .

(a) The class

$$
B^{-\infty, d}\left(\mathbb{R}_{+} ; N_{-}, N_{+}\right):=\bigcap_{m \in \mathbb{Z}} B^{m, d ; \lambda}\left(\mathbb{R}_{+} ; N_{-}, N_{+}\right)
$$

is seen to be independent of the type $\lambda$ of degeneracy. It is called the class of singular Green operators of type $d$. It coincides with the class $\Gamma^{d}\left(\mathbb{R}_{+} ; N_{-}, N_{+}\right)$considered in Schulze [20, Definition 4.1.76].

(b) Each singular Green operator $G: \mathcal{S}\left(\overline{\mathbb{R}}_{+}\right) \rightarrow \mathcal{S}\left(\overline{\mathbb{R}}_{+}\right)$of type $d$ can be uniquely represented in the form

$$
G u=\int_{0}^{\infty} \tilde{g}\left(t, t^{\prime}\right) u\left(t^{\prime}\right) d t^{\prime}+\sum_{k=0}^{d-1} \tilde{g}_{k}(t) \gamma_{k} u,
$$

where $\tilde{g} \in \mathcal{S}\left(\overline{\mathbb{R}}_{+} \times \overline{\mathbb{R}}_{+}\right), \tilde{g}_{k} \in \mathcal{S}\left(\overline{\mathbb{R}}_{+}\right), k=0,1, \ldots, d-1$, see Schulze [20, Proposition 4.1.46]. Similarly, each trace operator $S: \mathcal{S}\left(\overline{\mathbb{R}}_{+}\right) \rightarrow \mathbb{C}$ can be uniquely represented in the form

$$
S u=\int_{0}^{\infty} \tilde{s}\left(t^{\prime}\right) u\left(t^{\prime}\right) d t^{\prime}+\sum_{k=0}^{d-1} \tilde{s}_{k} \gamma_{k} u,
$$

where $\tilde{s} \in \mathcal{S}\left(\overline{\mathbb{R}}_{+}\right), \tilde{s}_{k} \in \mathbb{C}, k=0,1, \ldots, d-1$, see Schulze [20, Remark 4.1.77].

Proposition 3.6. Each $\mathfrak{A} \in B^{m, d ; \lambda}\left(\mathbb{R}_{+} ; N_{-}, N_{+}\right)$extends by continuity to an operator

$$
\begin{aligned}
& H^{s, \delta ; \lambda}\left(\mathbb{R}_{+}\right) \quad H^{s-m, \delta+m ; \lambda}\left(\mathbb{R}_{+}\right) \\
& \mathfrak{A}: \quad \oplus \quad \rightarrow \quad \oplus \\
& \mathbb{C}^{N_{-}} \quad \mathbb{C}^{N_{+}}
\end{aligned}
$$

for $s>d-1 / 2$. Furthermore, if $\mathfrak{A} \in B^{m-1, d ; \lambda}\left(\mathbb{R}_{+} ; N_{-}, N_{+}\right)$, then the operator in (3.4) is compact.

Proposition 3.7. For $\mathfrak{A} \in B^{m, d ; \lambda}\left(\mathbb{R}_{+} ; \tilde{N}, N_{+}\right), \mathfrak{B} \in B^{m^{\prime}, d^{\prime} ; \lambda}\left(\mathbb{R}_{+} ; N_{-}, \tilde{N}\right)$, composition yields

$$
\mathfrak{A} \mathfrak{B} \in B^{m+m^{\prime}, d^{\prime \prime} ; \lambda}\left(\mathbb{R}_{+} ; N_{-}, N_{+}\right),
$$

where $d^{\prime \prime}=\max \left\{d+m^{\prime}, d^{\prime}\right\}$.

Definition 3.8. If $\mathfrak{A} \in B^{m, d ; \lambda}\left(\mathbb{R}_{+} ; N_{-}, N_{+}\right)$is as in (3.3), then $\mathfrak{A}$ is called elliptic if

$$
\sigma_{\psi}^{m}(\mathfrak{A})(t, \tau):=\sigma_{\psi}^{m}\left(\mathrm{op}^{+}(a)\right)(t, \tau) \neq 0
$$

for all $(t, \tau) \in \overline{\mathbb{R}}_{+} \times(\mathbb{R} \backslash 0)$ and, in addition, there are constants $c>0, R>0$ such that

$$
|a(t, \tau)| \geq c\left|\tau, t^{l_{*}}\right|^{m}
$$

for all $(t, \tau) \in \overline{\mathbb{R}}_{+} \times \mathbb{R}$ with $\left|\tau, t^{l_{*}}\right| \geq R$.

Proposition 3.9. Let the operator $\mathfrak{A} \in B^{m, d ; \lambda}\left(\mathbb{R}_{+} ; N_{-}, N_{+}\right)$be elliptic. Then there is a parametrix $\mathfrak{B} \in B^{-m, 0 ; \lambda}\left(\mathbb{R}_{+} ; N_{+}, N_{-}\right)$to $\mathfrak{A}$, i.e., $\mathfrak{B}$ is so that

$$
\begin{aligned}
& \mathfrak{B} \mathfrak{A}-1 \in B^{-\infty, \max \{m, d\}}\left(\mathbb{R}_{+} ; N_{-}, N_{-}\right), \\
& \mathfrak{A} \mathfrak{B}-1 \in B^{-\infty,(d-m)^{+}}\left(\mathbb{R}_{+} ; N_{+}, N_{+}\right) .
\end{aligned}
$$


In particular, $\mathfrak{A}$ is a Fredholm operator when considered as operator in (3.4). There are finite-dimensional subspaces $L_{+} \subseteq \mathcal{S}\left(\overline{\mathbb{R}}_{+}\right) \oplus \mathbb{C}^{N_{-}}$and $L_{-} \subseteq \mathcal{S}\left(\overline{\mathbb{R}}_{+}\right) \oplus \mathbb{C}^{N_{+}}$independent of $s>d-1 / 2$ such that

$$
L_{+}=\operatorname{ker} \mathfrak{A}, \quad \operatorname{im} \mathfrak{A} \oplus L_{-}=H^{s-m, \delta+m ; \lambda}\left(\mathbb{R}_{+}\right) .
$$

The index ind $\mathfrak{A}=\operatorname{dim} L_{+}-\operatorname{dim} L_{-}$is then also independent of $s>d-1 / 2$.

Furthermore, $\mathfrak{A}$ is invertible as operator in (3.4) if and only if $\mathfrak{A}$ is invertible as operator in (3.3). In this case, $\mathfrak{A}^{-1} \in B^{-m,(d-m)^{+} ; \lambda}\left(\mathbb{R}_{+} ; N_{+}, N_{-}\right)$.

EXAmple 3.10. Let $A=\partial_{t}^{2}+2 i c t^{l_{*}} \partial_{t}-a t^{2 l_{*}}+i b t^{l_{*}-1}$, where $a, b, c \in \mathbb{R}$. Then ellipticity of $A \in B^{2,0 ; \lambda}\left(\mathbb{R}_{+}\right)$in the sense of Definition 3.8 is equivalent to

$$
c^{2}<a \text {. }
$$

In this case, ind $A=1$ by Remark 1.1. In fact, $\operatorname{dim}$ coker $A=0$, since it is seen that $A: \mathcal{S}\left(\overline{\mathbb{R}}_{+}\right) \rightarrow \mathcal{S}\left(\overline{\mathbb{R}}_{+}\right)$is surjective. Furthermore, $\operatorname{dim} \operatorname{ker} A=1$, since the O.D.E. $A u=0$ possesses a fundamental system $\left\{u_{+}, u_{-}\right\}$with the property that

$$
u_{ \pm}(t) \sim t^{\mu_{ \pm}} \exp \left( \pm \sqrt{a-c^{2}} \beta_{*} t^{l_{*}+1}\right) \quad \text { as } t \rightarrow \infty
$$

for certain $\mu_{ \pm} \in \mathbb{R}$, as asymptotic analysis shows, see, e.g., Olver [17]. Note that $u_{-}$is uniquely determined.

Now, given $a, c \in \mathbb{R}$, let $D_{a, c}$ be the set of all $b \in \mathbb{R}$ for which $u_{-}(0)=0$ holds. We conclude that the operator

$$
\left(\begin{array}{c}
\partial_{t}^{2}+i c t^{l_{*}} \partial_{t}-a t^{2 l_{*}}+i b t^{l_{*}-1} \\
\gamma_{0}
\end{array}\right) \in B^{2,1 ; \lambda}\left(\mathbb{R}_{+} ; 0,1\right)
$$

is invertible if and only if $b \notin D_{a, c}$ (i.e., $u_{-}(0) \neq 0$ ). Then its inverse belongs to $B^{-2,0 ; \lambda}\left(\mathbb{R}_{+} ; 1,0\right)$. It can be shown that $D_{a, c} \subset \mathbb{R}$ is a discrete set.

\section{The boundary symbolic calculus}

4.1. Estimates on interior symbols. The construction of the interior symbols is probably the most innovative part of the paper. An explanation for the kind of symbol estimates chosen in (4.1) has been given in Section 1. Another novelty of the approach lies in using functions possessing asymptotic expansions into double homogeneous components. This allows one to get control on both principal symbolic levels at a time. Comparable arguments in an analogous situation have been provided by Hirschmann [9]. Let us, in particular, emphasize the asymptotic summation results in Proposition 4.5.

Let $Z:=\left\{(t, x, \tau, \xi) \in \overline{\mathbb{R}}_{+} \times \Omega \times \mathbb{R}^{1+n} ;(t, \tau)=0\right.$ or $\left.(\tau, \xi)=0\right\}$.

Definition 4.1. Let $m, \eta \in \mathbb{Z}$.

(a) The symbol class $S^{m, \eta ; \lambda}\left([0, T) \times \Omega \times \mathbb{R}^{1+n}\right)$ consists of all $a \in C^{\infty}([0, T) \times \Omega \times$ $\left.\mathbb{R}^{1+n}\right)$ such that, for all $(j, k, \alpha, \beta) \in \mathbb{N}^{2+2 n}, 0<T^{\prime}<T$, and $K \Subset \Omega$, there is a constant $C_{j k \alpha \beta T^{\prime} K}>0$ such that

$$
\left|\partial_{t}^{k} \partial_{x}^{\beta} \partial_{\tau}^{j} \partial_{\xi}^{\alpha} a(t, x, \tau, \xi)\right| \leq C_{j k \alpha \beta T^{\prime} K} \Phi(t, \tau, \xi)^{m-j}\left(\langle\xi\rangle^{-\beta_{*}}+t\right)^{m-\eta-k}\langle\xi\rangle^{-|\alpha|}
$$

for all $(t, x, \tau, \xi) \in\left[0, T^{\prime}\right] \times K \times \mathbb{R}^{1+n}$. 
(b) The symbol class $S^{(\eta) ; \lambda}\left(\left(\overline{\mathbb{R}}_{+} \times \Omega \times \mathbb{R}^{1+n}\right) \backslash Z\right)$ consists of all $a \in C^{\infty}\left(\left(\overline{\mathbb{R}}_{+} \times \Omega \times\right.\right.$ $\left.\mathbb{R}^{1+n}\right) \backslash Z$ ) such that

$$
a\left(\nu^{-1} t, x, \nu \tau, \nu^{l_{*}+1} \xi\right)=\nu^{\eta} a(t, x, \tau, \xi), \quad \nu>0,
$$

for all $(t, x, \tau, \xi) \in\left(\overline{\mathbb{R}}_{+} \times \Omega \times \mathbb{R}^{1+n}\right) \backslash Z$.

(c) The symbol class $S^{(m, \eta) ; \lambda}\left(\mathbb{R}_{+} \times \Omega \times\left(\mathbb{R}^{1+n} \backslash 0\right)\right)$ consists of all $a \in C^{\infty}\left(\mathbb{R}_{+} \times \Omega \times\right.$ $\left.\left(\mathbb{R}^{1+n} \backslash 0\right)\right)$ such that

$$
a\left(\nu^{-1} t, x, \mu \nu \tau, \mu \nu^{l_{*}+1} \xi\right)=\mu^{m} \nu^{\eta} a(t, x, \tau, \xi), \quad \mu>0, \nu>0,
$$

for all $(t, x, \tau, \xi) \in \mathbb{R}_{+} \times \Omega \times\left(\mathbb{R}^{1+n} \backslash 0\right)$.

EXAmple 4.2. For $m \in \mathbb{N}, \eta \in \mathbb{Z}$, let

$$
a(t, x, \tau, \xi):=\sum_{j+|\alpha|=m} a_{j \alpha}(x) t^{m-\eta} \tau^{j}\left(t^{l *} \xi\right)^{\alpha},
$$

where $a_{j \alpha} \in C^{\infty}(\Omega)$ for $j+|\alpha|=m$. Then $a \in S^{(m, \eta) ; \lambda}\left(\mathbb{R}_{+} \times \Omega \times\left(\mathbb{R}^{1+n} \backslash 0\right)\right)$.

LEMmA 4.3. We have $S^{m, \eta ; \lambda}\left([0, T) \times \Omega \times \mathbb{R}^{1+n}\right) \subseteq S^{m^{\prime}, \eta^{\prime} ; \lambda}\left([0, T) \times \Omega \times \mathbb{R}^{1+n}\right)$ if and only if

$$
m \leq m^{\prime}, \quad \eta \leq \eta^{\prime}
$$

LEMma 4.4. For $a \in S^{(m, \eta) ; \lambda}\left(\mathbb{R}_{+} \times \mathbb{R}^{n} \times\left(\mathbb{R}^{1+n} \backslash 0\right)\right)$,

$$
\chi\left(\left|\tau, t^{l *} \xi\right|\right) a \in S^{m, \eta ; \lambda}\left([0, T) \times \Omega \times \mathbb{R}^{1+n}\right) .
$$

(Recall that $\chi$ is an excision function.)

Proof. We have

$$
a(t, x, \tau, \xi)=\left\langle\tau, t^{l_{*}} \xi\right\rangle^{m} t^{m-\eta} a\left(1, x, \frac{\tau}{\left\langle\tau, t^{l_{*}} \xi\right\rangle}, \frac{t^{l_{*}} \xi}{\left\langle\tau, t^{l_{*}} \xi\right\rangle}\right)
$$

as well as $\Phi(t, \tau, \xi) \sim\left\langle\tau, t^{l_{*}} \xi\right\rangle,\langle\xi\rangle^{-\beta_{*}}+t \sim t$ when $t^{l_{*}+1}\langle\xi\rangle \geq 1$, see Remark 1.3. Furthermore, we have

$$
a(t, x, \tau, \xi)=\left(|\tau|+\langle\xi\rangle^{\beta_{*}}\right)^{m}\langle\xi\rangle^{-\beta_{*}(m-\eta)} a\left(t\langle\xi\rangle^{\beta_{*}}, x, \frac{\tau}{|\tau|+\langle\xi\rangle^{\beta_{*}}}, \frac{\langle\xi\rangle^{-\beta_{*} l_{*}} \xi}{|\tau|+\langle\xi\rangle^{\beta_{*}}}\right)
$$

and $t\langle\xi\rangle^{\beta_{*}} \leq 1, \Phi(t, \tau, \xi) \sim|\tau|+\langle\xi\rangle^{\beta_{*}},\langle\xi\rangle^{-\beta_{*}}+t \sim\langle\xi\rangle^{-\beta_{*}}$ when $t^{l_{*}+1}\langle\xi\rangle \leq 1$, see again Remark 1.3. This yields

$$
\left|\chi\left(\left|\tau, t^{l_{*}} \xi\right|\right) a\right| \leq C \Phi(t, \tau, \xi)^{m}\left(\langle\xi\rangle^{-\beta_{*}}+t\right)^{m-\eta}
$$

for some constant $C>0$. Moreover, $\partial_{t}^{k} \partial_{x}^{\beta} \partial_{\tau}^{j} \partial_{\xi}^{\alpha} a \in S^{\left(m-j-|\alpha|, \eta-j+k-\left(l_{*}+1\right)|\alpha|\right) ; \lambda}\left(\mathbb{R}_{+} \times \Omega \times\right.$ $\left.\left(\mathbb{R}^{1+n} \backslash 0\right)\right)$ for all $j, k, \alpha, \beta$ yielding

$$
\left|\partial_{t}^{k} \partial_{x}^{\beta} \partial_{\tau}^{j} \partial_{\xi}^{\alpha}\left(\chi\left(\left|\tau, t^{l_{*}} \xi\right|\right) a\right)\right| \leq C \Phi(t, \tau, \xi)^{m-j-|\alpha|}\left(\langle\xi\rangle^{-\beta_{*}}+t\right)^{m-\eta-k+l_{*}|\alpha|},
$$

since derivatives on $\chi\left(\left|\tau, t^{l_{*}} \xi\right|\right)$ are harmless. Taking into account that

$$
\Phi(t, \tau, \xi)^{-|\alpha|}\left(\langle\xi\rangle^{-\beta_{*}}+t\right)^{l_{*}|\alpha|} \leq \Phi(t, 0, \xi)^{-|\alpha|}\left(\langle\xi\rangle^{-\beta_{*}}+t\right)^{l_{*}|\alpha|} \sim\langle\xi\rangle^{-|\alpha|}
$$

concludes the proof. 
There are two different possibilities of forming asymptotic sums in the symbol classes $S^{m, \eta ; \lambda}\left([0, T) \times \Omega \times \mathbb{R}^{1+n}\right)$ for $m, \eta \in \mathbb{Z}$; one with leaving $\eta$ constant, the other one with leaving $m$ constant, cf. Lemma 4.3 .

Proposition 4.5. Let $m, \eta \in \mathbb{Z}$.

(a) Let $a_{j} \in S^{m-j, \eta ; \lambda}\left([0, T) \times \Omega \times \mathbb{R}^{1+n}\right)$ for $j=0,1,2, \ldots$ Then there is an a in $S^{m, \eta ; \lambda}\left([0, T) \times \Omega \times \mathbb{R}^{1+n}\right)$ such that, for all $J \in \mathbb{N}$,

$$
a-\sum_{j<J} a_{j} \in S^{m-J, \eta ; \lambda}\left([0, T) \times \Omega \times \mathbb{R}^{1+n}\right) .
$$

Moreover, a is uniquely determined modulo $\bigcap_{j \in \mathbb{N}} S^{m-j, \eta ; \lambda}\left([0, T) \times \Omega \times \mathbb{R}^{1+n}\right)$.

(b) Let $\tilde{a}_{k} \in S^{m, \eta-k ; \lambda}\left([0, T) \times \Omega \times \mathbb{R}^{1+n}\right)$ for $k=0,1,2, \ldots$ Then there is an $\tilde{a}$ in $S^{m, \eta ; \lambda}\left([0, T) \times \Omega \times \mathbb{R}^{1+n}\right)$ such that, for all $K \in \mathbb{N}$,

$$
\tilde{a}-\sum_{k<K} \tilde{a}_{k} \in S^{m, \eta-K ; \lambda}\left([0, T) \times \Omega \times \mathbb{R}^{1+n}\right) .
$$

Moreover, $\tilde{a}$ is uniquely determined modulo $\bigcap_{k \in \mathbb{N}} S^{m, \eta-k ; \lambda}\left([0, T) \times \Omega \times \mathbb{R}^{1+n}\right)$.

Proof. (a) The symbol $a$ is found in the form

$$
a=\sum_{j=0}^{\infty}\left(1-\kappa\left(c_{j}^{-1} \tau, c_{j}^{-\left(l_{*}+1\right)} \xi\right)\left(1-\chi\left(c_{j}^{-1} t^{l_{*}+1}\langle\xi\rangle\right)\right)\right) a_{j},
$$

for some sequence $\left\{c_{j}\right\} \subset \mathbb{R}_{+}$with $c_{j} \rightarrow \infty$ as $j \rightarrow \infty$ sufficiently fast, where the function $\kappa \in C^{\infty}\left(\mathbb{R}^{1+n}\right)$ is chosen to possess the following properties:

$$
\begin{gathered}
\kappa\left(\mu \tau, \mu^{l_{*}+1} \xi\right)=\kappa(\tau, \xi), \quad \mu \geq 1,|\tau|+|\xi|^{\beta_{*}} \geq 1, \\
\kappa(\tau, \xi)= \begin{cases}0 & \text { if }|\tau|+|\xi|^{\beta_{*}} \leq 1 / 2 \\
0 & \text { if }|\tau|+|\xi|^{\beta_{*}} \geq 1,|\tau| \geq|\xi|^{\beta_{*}} \\
1 & \text { if }|\tau|+|\xi|^{\beta_{*}} \geq 1,|\tau| \leq(1 / 2)|\xi|^{\beta_{*}} .\end{cases}
\end{gathered}
$$

(In general, the sequence $\left\{c_{j}\right\}$ depends on the $a_{j}$.) The proof relies on the following facts:

- $\kappa(\tau, \xi)\left(1-\chi\left(t^{l_{*}+1}\langle\xi\rangle\right)\right) \in \bigcap_{j \in \mathbb{N}} S^{-j, 0 ; \lambda}\left([0, T) \times \Omega \times \mathbb{R}^{1+n}\right)$;

- $\left\{1-\kappa\left(c^{-1} \tau, c^{-\left(l_{*}+1\right)} \xi\right)\left(1-\chi\left(c^{-1} t^{l_{*}+1}\langle\xi\rangle\right)\right) ; c \geq 1\right\} \subset S^{0,0 ; \lambda}\left([0, T) \times \Omega \times \mathbb{R}^{1+n}\right)$ is a bounded subset;

- for some $C>0$,

$$
\begin{aligned}
& \sup _{|\tau|+t^{l_{*}+1}\langle\xi\rangle \geq c} \frac{\left(\langle\xi\rangle^{-\beta_{*}}+t\right)^{-1}}{\Phi(t, \tau, \xi)} \\
& \quad \leq C \sup _{|\tau|+t^{l_{*}+1}\langle\xi\rangle \geq c} \min \left\{\frac{\langle\xi\rangle^{\beta_{*}}}{|\tau|+\langle\xi\rangle^{\beta_{*}}}, \frac{1}{1+t^{l_{*}+1}\langle\xi\rangle}\right\} \rightarrow 0 \quad \text { as } c \rightarrow \infty .
\end{aligned}
$$

(b) Now, the symbol $\tilde{a}$ is found in the form

$$
\tilde{a}=\sum_{k=0}^{\infty}\left(1-\chi\left(c_{k} t\right)\right) \chi\left(c_{k}^{-1}|\xi|\right) \tilde{a}_{k}
$$


for some sequence $\left\{c_{k}\right\} \subset \mathbb{R}_{+}$with $c_{k} \rightarrow \infty$ as $k \rightarrow \infty$ sufficiently fast. This time, the proof relies on the following facts:

- $1-(1-\chi(t)) \chi(|\xi|) \in \bigcap_{k \in \mathbb{N}} S^{0,-k ; \lambda}\left([0, T) \times \Omega \times \mathbb{R}^{1+n}\right)$ because of $\chi(t), 1-\chi(|\xi|) \in$ $\bigcap_{k \in \mathbb{N}} S^{0,-k ; \lambda}\left([0, T) \times \Omega \times \mathbb{R}^{1+n}\right)$.

- $\left\{(1-\chi(c t)) \chi\left(c^{-1}|\xi|\right) ; c \geq 1\right\} \subset S^{0,0 ; \lambda}\left([0, T) \times \Omega \times \mathbb{R}^{1+n}\right)$ is a bounded subset.

- $\sup _{|\xi| \geq c, t \leq c^{-1}}\left(\langle\xi\rangle^{-\beta_{*}}+t\right) \rightarrow 0$ as $c \rightarrow \infty$.

For a general scheme of performing asymptotic sums, see Schulze [20, Prop. 1.1.17].

In both cases (a) and (b), asymptotic summation can be performed better if one has additional information concerning the $a_{j}$ and $\tilde{a}_{k}$, respectively.

Proposition 4.6.

(a) In Proposition 4.5 (a), if the $a_{j}$ for $j=0,1, \ldots$ satisfy

$$
a_{j}\left(\nu^{-1} t, x, \mu \nu \tau, \mu \nu^{l_{*}+1} \xi\right)=\mu^{m-j} \nu^{\eta} a_{j}(t, x, \tau, \xi), \quad \mu \geq 1, \nu \geq 1,
$$

when $\left|\tau, t^{l_{*}} \xi\right| \geq 1$, then the symbol $a \in S^{m, \eta ; \lambda}\left([0, T) \times \Omega \times \mathbb{R}^{1+n}\right)$ can be chosen to satisfy

$$
a\left(\nu^{-1} t, x, \nu \tau, \nu^{l_{*}+1} \xi\right)=\nu^{\eta} a(t, x, \tau, \xi), \quad \nu \geq 1,
$$

when $\left|\tau, t^{l_{*}} \xi\right| \geq 1$.

(b) In Proposition $4.5(\mathrm{~b})$, if the $\tilde{a}_{k}$ for $k=0,1, \ldots$ satisfy

$$
\tilde{a}_{k}\left(\nu^{-1} t, x, \mu \nu \tau, \mu \nu^{l_{*}+1} \xi\right)=\mu^{m} \nu^{\eta-k} \tilde{a}_{k}(t, x, \tau, \xi), \quad \mu \geq 1, \nu \geq 1,
$$

when $\left|\tau, t^{l_{*}} \xi\right| \geq 1$, then the symbol $\tilde{a} \in S^{m, \eta ; \lambda}\left([0, T) \times \Omega \times \mathbb{R}^{1+n}\right)$ can be chosen to satisfy

$$
\tilde{a}(t, x, \mu \tau, \mu \xi)=\mu^{m} \tilde{a}(t, x, \tau, \xi), \quad \mu \geq 1,
$$

when $\left|\tau, t^{l_{*}} \xi\right| \geq 1$.

We only prove (a) (after Lemma 4.9); the proof of (b) is similar, upon replacing in the following argument the set $\left\{(t, x, \tau, \xi) \in \mathbb{R}_{+} \times \Omega \times\left(\mathbb{R}^{1+n} \backslash 0\right) ;\left|\tau, t^{l_{*}} \xi\right|=1\right\}$ by the set $\left\{(t, x, \tau, \xi) \in \mathbb{R}_{+} \times \Omega \times\left(\mathbb{R}^{1+n} \backslash 0\right) ;|\tau, \xi|=1\right\}$ and treating the behaviour of symbols on this set as $t \rightarrow+0$.

To prepare for the proof of (a), we state:

LEMMA 4.7.

(a) A function $a \in S^{(\eta) ; \lambda}\left(\left(\overline{\mathbb{R}}_{+} \times \Omega \times \mathbb{R}^{1+n}\right) \backslash Z\right)$ is determined, for each fixed $x \in \Omega$, by its values on the hypersurface

$$
\Theta:=\left\{(t, \tau, \xi) \in \overline{\mathbb{R}}_{+} \times\left(\mathbb{R}^{1+n} \backslash 0\right) ;\left|\tau, t^{l_{*}} \xi\right|=1\right\} .
$$

A function $a \in S^{(m, \eta) ; \lambda}\left(\mathbb{R}_{+} \times \Omega \times\left(\mathbb{R}^{1+n} \backslash 0\right)\right)$ is determined, for each fixed $x \in \Omega$, by its values on $\Theta_{1}:=\left\{(1, \tau, \xi) \in \overline{\mathbb{R}}_{+} \times\left(\mathbb{R}^{1+n} \backslash 0\right) ;|\tau, \xi|=1\right\}$.

(b) Given a function $b \in C^{\infty}\left(\Omega ; C^{\infty}(\Theta)\right)$, its homogeneous extension according to (4.12) below belongs to $S^{(m, \eta) ; \lambda}\left(\mathbb{R}_{+} \times \Omega \times\left(\mathbb{R}^{1+n} \backslash 0\right)\right)$ if and only if

$$
b(t, x, \tau, \xi)=t^{m-\eta} b\left(1, x, \tau, t^{l_{*}} \xi\right), \quad\left|\tau, t^{l_{*}} \xi\right|=1 .
$$


Proof. In fact, we have

$$
a(t, x, \tau, \xi)=\left|\tau, t^{l_{*}} \xi\right|^{\eta} a\left(\left|\tau, t^{l_{*}} \xi\right| t, x, \frac{\tau}{\left|\tau, t^{l_{*}} \xi\right|}, \frac{\xi}{\left|\tau, t^{l_{*}} \xi\right|^{l_{*}+1}}\right)
$$

when $a \in S^{(\eta) ; \lambda}\left(\left(\overline{\mathbb{R}}_{+} \times \Omega \times \mathbb{R}^{1+n}\right) \backslash Z\right)$ and

$$
a(t, x, \tau, \xi)=\left|\tau, t^{l_{*}} \xi\right|^{m} t^{m-\eta} a\left(1, x, \frac{\tau}{\mid \tau, t_{*}^{l_{*} \xi \mid}}, \frac{t^{l_{*}} \xi}{\mid \tau, t^{l_{*} \xi \mid}}\right)
$$

when $a \in S^{(m, \eta) ; \lambda}\left(\mathbb{R}_{+} \times \Omega \times\left(\mathbb{R}^{1+n} \backslash 0\right)\right)$.

Note that relation (4.11) only depends on the difference $m-\eta$.

By virtue of $(4.11)$, the embedding $S^{(m, \eta) ; \lambda}\left(\mathbb{R}_{+} \times \Omega \times\left(\mathbb{R}^{1+n} \backslash 0\right)\right) \subset S^{(\eta) ; \lambda}\left(\left(\overline{\mathbb{R}}_{+} \times\right.\right.$ $\left.\Omega \times \mathbb{R}^{1+n}\right) \backslash Z$ ) to hold requires that $\eta \leq m$, which is far from the situation we are interested in, cf. (4.4). For functions $b \in C^{\infty}\left(\Omega ; C^{\infty}(\Theta)\right)$, we can, however, admit asymptotic expansions into homogeneous components when $t \geq 1$ : To do so, consider the vector fields

$$
\left\{\begin{aligned}
V_{0} & =t \partial_{t}-l_{*} \xi \cdot \partial_{\xi}, & & \\
V_{j} & =t^{l_{*}} \xi_{j} \partial_{\tau}-t^{-l_{*}} \tau \partial_{\xi_{j}}, & & j=1, \ldots, n, \\
V_{i j} & =\xi_{i} \partial_{\xi_{j}}-\xi_{j} \partial_{\xi_{i}}, & & 1 \leq i<j \leq n,
\end{aligned}\right.
$$

on $\mathbb{R}_{+} \times \Omega \times\left(\mathbb{R}^{1+n} \backslash 0\right)$. These vector fields generate the Lie algebra of all vector fields tangent to $\left\{(t, x, \tau, \xi) \in \mathbb{R}_{+} \times \Omega \times\left(\mathbb{R}^{1+n} \backslash 0\right) ;(t, \tau, \xi) \in \Theta\right\}$ over the smooth functions. Furthermore, these vector fields map the spaces $S^{(m, \eta) ; \lambda}\left(\mathbb{R}_{+} \times \Omega \times\left(\mathbb{R}^{1+n} \backslash 0\right)\right)$ for $m, \eta \in \mathbb{Z}$ into themselves.

Compare the following definition to (4.11).

Definition 4.8. Define $\Upsilon^{\tilde{\eta}}$ for $\tilde{\eta} \in \mathbb{Z}$ to be the space of all $b \in C^{\infty}\left(\Omega ; C^{\infty}(\Theta)\right)$ for which, for all $\gamma \in \mathbb{N}^{1+n+}\left(\begin{array}{c}n \\ 2\end{array}\right)$ and $K \Subset \Omega$, there is a constant $C_{\gamma K}>0$ such that

$$
\left|V_{0}^{\gamma_{0}} V_{1}^{\gamma_{1}} \ldots V_{n-1, n}^{\gamma_{n-1, n}} b(t, x, \tau, \xi)\right| \leq C_{\gamma K} t^{-\tilde{\eta}}
$$

for all $t \geq 1, x \in K$, and $(t, \tau, \xi) \in \Theta$.

Asymptotic summation in the spaces $\Upsilon^{\tilde{\eta}}$ is possible:

LEMmA 4.9. If $b_{k} \in \Upsilon^{\tilde{\eta}+k}$ for $k=0,1,2, \ldots$ are given, then there is $a b \in \Upsilon^{\tilde{\eta}}$ such that, for all $K \in \mathbb{N}$,

$$
b-\sum_{k<K} b_{k} \in \Upsilon^{\tilde{\eta}+K} .
$$

Proof of Proposition 4.6 (a). Take the $a_{j}$ as in Proposition 4.5 (a) and assume that (4.9) is fulfilled. Let $\bar{a}_{j}$ be the homogenization of $a_{j}$ according to (4.13) (with $(m, \eta)$ replaced by $(m-j, \eta))$ and $b_{j}$ be the restriction of $\bar{a}_{j}$ to $\left\{(t, x, \tau, \xi) \in \mathbb{R}_{+} \times \Omega \times\left(\mathbb{R}^{1+n} \backslash 0\right)\right.$; $(t, \tau, \xi) \in \Theta\}$. Then $b_{j} \in \Upsilon^{-m+\eta+j}$ for $j=0,1,2, \ldots$ Choose $b \in \Upsilon^{-m+\eta}$ according to (4.14) and let $\bar{a}$ be its homogeneous extension according to (4.12).

Then the function $a(t, x, \tau, \xi)=\chi\left(\left|\tau, t^{l} \xi \xi\right|\right) \tilde{a}(t, x, \tau, \xi)$ has the desired properties.

Now, we are able to introduce the classes $S_{\mathrm{cl}}^{m, \eta ; \lambda}\left([0, T) \times \Omega \times \mathbb{R}^{1+n}\right)$ of symbols possessing asymptotic expansions into double homogeneous components. 
Definition 4.10. Let $m, \eta \in \mathbb{Z}$.

(a) $S^{[m], \eta ; \lambda}\left([0, T) \times \Omega \times \mathbb{R}^{1+n}\right)$ is the space of all $a \in S^{m, \eta ; \lambda}\left([0, T) \times \Omega \times \mathbb{R}^{1+n}\right)$ for which, for all $0<T^{\prime}<T, K \Subset \Omega$, there is a constant $c_{T^{\prime} K}>0$ such that $a(t, x, \mu \tau, \mu \xi)=$ $\mu^{m} a(t, x, \tau, \xi)$ whenever $\mu \geq 1,(t, x, \tau, \xi) \in[0, T) \times K \times \mathbb{R}^{1+n}$, and $\left|\tau, t^{l_{*}} \xi\right| \geq c_{T^{\prime} K}$.

(b) $S^{m,[\eta] ; \lambda}\left([0, T) \times \Omega \times \mathbb{R}^{1+n}\right)$ is the space of all $a \in S^{m, \eta ; \lambda}\left([0, T) \times \Omega \times \mathbb{R}^{1+n}\right)$ for which, for all $0<T^{\prime}<T, K \Subset \Omega$, there is a constant $c_{T^{\prime} K}>0$ such that $a\left(\nu^{-1} t, x, \nu \tau, \nu^{l_{*}+1} \xi\right)=\nu^{\eta} a(t, x, \tau, \xi)$ whenever $\nu \geq 1,(t, x, \tau, \xi) \in\left[0, T^{\prime}\right] \times K \times \mathbb{R}^{1+n}$, and $\left|\tau, t^{l} \xi\right| \geq c_{T^{\prime} K}$.

(c) $S_{\mathrm{cl}}^{m, \eta ; \lambda}\left([0, T) \times \Omega \times \mathbb{R}^{1+n}\right)$ is the space of all $a \in S^{m, \eta ; \lambda}\left([0, T) \times \Omega \times \mathbb{R}^{1+n}\right)$ for which there are functions $a_{j} \in S^{[m-j], \eta ; \lambda}\left([0, T) \times \Omega \times \mathbb{R}^{1+n}\right)$ for $j=0,1,2, \ldots$ and $\tilde{a}_{k} \in S^{m,[\eta-k] ; \lambda}\left([0, T) \times \Omega \times \mathbb{R}^{1+n}\right)$ for $k=0,1,2, \ldots$ with the following properties:

- For all $J \in \mathbb{N}$,

$$
a-\sum_{j<J} a_{j} \in S^{[m-J], \eta ; \lambda}\left([0, T) \times \Omega \times \mathbb{R}^{1+n}\right) .
$$

- For all $K \in \mathbb{N}$,

$$
a-\sum_{k<K} \tilde{a}_{k} \in S^{m,[\eta-K] ; \lambda}\left([0, T) \times \Omega \times \mathbb{R}^{1+n}\right) .
$$

- There are symbols $a_{j k} \in S^{[m-j], \eta-k ; \lambda}\left([0, T) \times \Omega \times \mathbb{R}^{1+n}\right) \cap S^{m-j,[\eta-k] ; \lambda}([0, T) \times$ $\left.\Omega \times \mathbb{R}^{1+n}\right)$ for $j, k=0,1,2, \ldots$ such that, for all $J, K \in \mathbb{N}$,

$$
\begin{gathered}
a_{j}-\sum_{k<K} a_{j k} \in S^{[m-j], \eta-K ; \lambda}\left([0, T) \times \Omega \times \mathbb{R}^{1+n}\right), \\
\tilde{a}_{k}-\sum_{j<J} a_{j k} \in S^{m-J,[\eta-k] ; \lambda}\left([0, T) \times \Omega \times \mathbb{R}^{1+n}\right) .
\end{gathered}
$$

In the situation described in (c), we write

$$
a \sim \sum_{j, k \in \mathbb{N}} a_{j k}
$$

and call this a double asymptotic expansion for $a$.

REmark 4.11. The requirements in Definition 4.10 (c) can be relaxed (then still defining the same symbol class); for a thorough discussion in an analogous situation, see Hirschmann [9].

Definition 4.12. For $a \in S_{\mathrm{cl}}^{m, \eta ; \lambda}\left([0, T) \times \Omega \times \mathbb{R}^{1+n}\right)$ as in the previous definition and $j, k \in \mathbb{N}$, we introduce:

- $\sigma_{\psi}^{m-j}(a)(t, x, \tau, \xi)$ for $(\tau, \xi) \neq 0$ is the homogenization of $a_{j}$;

- $\sigma_{b}^{\eta-k ; \lambda}(a)(t, x, \tau, \xi)$ for $(t, \tau, \xi) \notin Z$ is the homogenization of $\tilde{a}_{k}$;

- $\sigma_{\psi, b}^{m-j, \eta-k ; \lambda}(a)(t, x, \tau, \xi)$ for $t>0,(\tau, \xi) \neq 0$ is the (double) homogenization of $a_{j k}$.

For $a \in S_{\mathrm{cl}}^{m, \eta ; \lambda}\left([0, T) \times \Omega \times \mathbb{R}^{1+n}\right)$, all these symbols are obviously well-defined. By construction, a compatibility condition between the symbols $\sigma_{\psi}^{m}(a)(t, x, \tau, \xi)$ and 
$\sigma_{b}^{\eta ; \lambda}(a)(t, x, \tau, \xi)$ occurs:

$$
\lim _{\nu \rightarrow \infty} \nu^{-\eta} \sigma_{\psi}^{m}(a)\left(\nu^{-1} t, x, \nu \tau, \nu^{l_{*}+1} \xi\right)=\lim _{\mu \rightarrow \infty} \mu^{-m} \sigma_{b}^{\eta ; \lambda}(a)(t, x, \mu \tau, \mu \xi),
$$

both limits actually being equal to $\sigma_{\psi, b}^{m, \eta ; \lambda}(a)(t, x, \tau, \xi)$.

LEMmA 4.13. Let $m=\eta$ (this being the case we are interested in later on). Then there is a symbol $\tilde{\sigma}_{\psi}^{m ; \lambda}(a) \in S^{(m)}\left([0, T) \times \Omega \times\left(\mathbb{R}^{1+n} \backslash 0\right)\right)$ (i.e., it is smooth up to $t=0$ ) such that

$$
\sigma_{\psi}^{m}(a)(t, x, \tau, \xi)=\tilde{\sigma}_{\psi}^{m ; \lambda}(a)\left(t, x, \tau, t^{l_{*}} \xi\right) .
$$

Utilizing this symbol, the compatibility condition (4.16) is expressed as follows:

$$
\sigma_{\psi}^{m}\left(\sigma_{b}^{m ; \lambda}(a)\right)(t, x, \tau, \xi)=\tilde{\sigma}_{\psi}^{m ; \lambda}(a)\left(0, x, \tau, t^{l_{*}} \xi\right) .
$$

Proof. From (4.16), it is seen that the limit

$$
\begin{aligned}
\tilde{\sigma}_{\psi}^{m ; \lambda}(a)(0, x, \tau, \tilde{\xi}):=\lim _{t \rightarrow+0} & \sigma_{\psi}^{m}(a)\left(t, x, \tau, t^{-l_{*}} \tilde{\xi}\right) \\
& =\lim _{t \rightarrow+0} t^{m} \sigma_{\psi}^{m}(a)\left(t, x, t^{-1} \tau, t^{-\left(l_{*}+1\right)} \tilde{\xi}\right)=\sigma_{\psi, b}^{m, m ; \lambda}(1, x, \tau, \tilde{\xi})
\end{aligned}
$$

exists. Furthermore, the left-hand side of (4.17) equals the right-hand side of (4.16), and this, in turn, is equal to

$$
\sigma_{\psi, b}^{m, m ; \lambda}(a)(t, x, \tau, \xi)=\sigma_{\psi, b}^{m, m ; \lambda}\left(1, x, \tau, t^{l *} \xi\right)
$$

by $(4.13)$.

For later reference, we also denote by $S_{\mathrm{cl}}^{m,(\eta) ; \lambda}\left(\left(\overline{\mathbb{R}}_{+} \times \Omega \times \mathbb{R}^{1+n}\right) \backslash Z\right)$ the space of all homogenizations of functions $\tilde{a}_{0}$ according to Definition 4.12 .

REMARK 4.14. For $a \in S_{\mathrm{cl}}^{m, \eta ; \lambda}\left([0, T) \times \Omega \times \mathbb{R}^{1+n}\right)$,

$$
a \in S_{\mathrm{cl}}^{m-1, \eta-1 ; \lambda}\left([0, T) \times \Omega \times \mathbb{R}^{1+n}\right) \Longleftrightarrow \sigma_{\psi}^{m}(a)=0, \sigma_{b}^{\eta ; \lambda}(a)=0 .
$$

This leads to a short exact split sequence, see also Proposition 4.21.

4.2. Symbols with the $\lambda$-transmission property. To ensure the mapping properties in (1.8) (i.e., to preserve smoothness up to $t=0$ ), the transmission property in an appropriate form is to be added to the calculus.

The following definition is motivated by the standard situation, see (2.2):

Definition 4.15. A symbol $a \in S^{m, m ; \lambda}\left([0, T) \times \Omega \times \mathbb{R}^{1+n}\right)$ is said to possess the $\lambda$-transmission property if

$$
\partial_{t}^{k} a\left(0, x,\langle\xi\rangle^{\beta_{*}} \tau, \xi\right) \in S^{\beta_{*}(m+k)}\left(\Omega \times \mathbb{R}^{n} ; H_{m+1}\right)
$$

for all $k=0,1,2, \ldots$

The following proposition is proven in a standard manner:

Proposition 4.16. A symbol $a \in S_{\mathrm{cl}}^{m, m ; \lambda}\left([0, T) \times \Omega \times \mathbb{R}^{1+n}\right)$ possesses the $\lambda$-transmission property if and only if

$$
\partial_{t}^{k} \partial_{\xi}^{\alpha} \sigma_{b}^{m-j ; \lambda}(a)(0, x,-1,0)=(-1)^{m-j-\left(l_{*}+1\right)|\alpha|+k} \partial_{t}^{k} \partial_{\xi}^{\alpha} \sigma_{b}^{m-j ; \lambda}(a)(0, x, 1,0)
$$

for all $j, k \in \mathbb{N}, \alpha \in \mathbb{N}^{n}$. 
In particular, it makes sense to say that a symbol $a \in S^{(m) ; \lambda}\left(\left(\overline{\mathbb{R}}_{+} \times \Omega \times \mathbb{R}^{1+n}\right) \backslash Z\right)$ satisfying (4.18) with $j=0$ possesses $\lambda$-transmission property.

We eventually introduce our basic symbol classes:

Definition 4.17. For $m \in \mathbb{Z}$, the symbol class $S_{\mathrm{tr}, \mathrm{cl}}^{m ; \lambda}\left([0, T) \times \Omega \times \mathbb{R}^{1+n}\right)$ consists of all functions

$$
a \in S_{\mathrm{cl}}^{m, m ; \lambda}\left([0, T) \times \Omega \times \mathbb{R}^{1+n}\right) \cap S_{\mathrm{tr}, \mathrm{cl}}^{m}\left([0, T) \times \Omega \times \mathbb{R}^{1+n}\right)
$$

possessing the $\lambda$-transmission property.

We have $S_{\mathrm{tr}, \mathrm{cl}}^{m-1 ; \lambda}\left([0, T) \times \Omega \times \mathbb{R}^{1+n}\right) \subseteq S_{\mathrm{tr}, \mathrm{cl}}^{m ; \lambda}\left([0, T) \times \Omega \times \mathbb{R}^{1+n}\right)$ and

$$
\bigcap_{m \in \mathbb{Z}} S_{\mathrm{tr}, \mathrm{cl}}^{m ; \lambda}\left([0, T) \times \Omega \times \mathbb{R}^{1+n}\right)=C^{\infty}\left([0, T) \times \Omega ; \mathcal{S}\left(\mathbb{R}^{1+n}\right)\right) .
$$

Proposition 4.18. Let $a_{j} \in S_{\mathrm{tr}, \mathrm{cl}}^{m-j ; \lambda}\left([0, T) \times \Omega \times \mathbb{R}^{1+n}\right)$ for $j=0,1,2, \ldots$ Then there is a symbol $a \in S_{\mathrm{tr}, \mathrm{cl}}^{m ; \lambda}\left([0, T) \times \Omega \times \mathbb{R}^{1+n}\right)$ such that, for all $J \in \mathbb{N}$,

$$
a-\sum_{j<J} a_{j} \in S_{\mathrm{tr}, \mathrm{cl}}^{m-J}\left([0, T) \times \Omega \times \mathbb{R}^{1+n}\right) .
$$

Proposition 4.19. For the symbol classes $S_{\mathrm{tr}, \mathrm{cl}}^{m ; \lambda}\left([0, T) \times \Omega \times \mathbb{R}^{1+n}\right)$ for $m \in \mathbb{Z}$ the following rules hold:

(a) $\partial_{t}^{k} \partial_{x}^{\beta} \partial_{\tau}^{j} \partial_{\xi}^{\alpha} a \in S_{\mathrm{tr}, \mathrm{cl}}^{m-j-|\alpha| ; \lambda}\left([0, T) \times \Omega \times \mathbb{R}^{1+n}\right)$ if $a \in S_{\mathrm{tr}, \mathrm{cl}}^{m ; \lambda}\left([0, T) \times \Omega \times \mathbb{R}^{1+n}\right)$.

(b) $a b \in S_{\mathrm{tr}, \mathrm{cl}}^{m+m^{\prime} ; \lambda}\left([0, T) \times \Omega \times \mathbb{R}^{1+n}\right)$ if $a \in S_{\mathrm{tr}, \mathrm{cl}}^{m ; \lambda}\left([0, T) \times \Omega \times \mathbb{R}^{1+n}\right), b \in S_{\mathrm{tr}, \mathrm{cl}}^{m^{\prime} ; \lambda}([0, T) \times$ $\left.\Omega \times \mathbb{R}^{1+n}\right)$. Furthermore,

$$
\sigma_{\psi}^{m+m^{\prime}}(a b)=\sigma_{\psi}^{m}(a) \sigma_{\psi}^{m^{\prime}}(b), \quad \sigma_{b}^{m+m^{\prime} ; \lambda}(a b)=\sigma_{b}^{m ; \lambda}(a) \sigma_{b}^{m^{\prime} ; \lambda}(b) .
$$

(c) $a \circ b \in S_{\mathrm{tr}, \mathrm{cl}}^{m+m^{\prime} ; \lambda}\left([0, T) \times \Omega \times \mathbb{R}^{1+n}\right)$ if $a \in S_{\mathrm{tr}, \mathrm{cl}}^{m ; \lambda}\left([0, T) \times \Omega \times \mathbb{R}^{1+n}\right), b \in S_{\mathrm{tr}, \mathrm{cl}}^{m^{\prime} ; \lambda}([0, T) \times$ $\left.\Omega \times \mathbb{R}^{1+n}\right)$, where "о" is the Leibniz product. Furthermore,

$$
(a \circ b)(t, x, \tau, \xi) \sim \sum_{j, \alpha} \frac{1}{j ! \alpha !} \partial_{\tau}^{j} \partial_{\xi}^{\alpha} a(t, x, \tau, \xi) D_{t}^{j} D_{x}^{\alpha} b(t, x, \tau, \xi) .
$$

Concerning the next definition, see Lemma 4.13.

Definition 4.20. The principal symbol space $\Sigma S_{\mathrm{tr}, \mathrm{cl}}^{m ; \lambda}\left([0, T) \times \Omega \times \mathbb{R}^{1+n}\right)$ consists of all couples $(a, \alpha) \in S_{\mathrm{tr}}^{(m)}\left([0, T) \times \Omega \times\left(\mathbb{R}^{1+n} \backslash 0\right)\right) \times S_{\mathrm{cl}}^{m,(m) ; \lambda}\left(\left(\overline{\mathbb{R}}_{+} \times \Omega \times \mathbb{R}^{1+n}\right) \backslash Z\right)$, where $\alpha$ possesses the $\lambda$-transmission property,

$$
a(t, x, \tau, \xi)=\tilde{a}\left(t, x, \tau, t^{l_{*}} \xi\right)
$$

for some $\tilde{a} \in S^{(m)}\left([0, T) \times \Omega \times\left(\mathbb{R}^{1+n} \backslash 0\right)\right)$, and

$$
\sigma_{\psi}^{m}(\alpha)(t, x, \tau, \xi)=\tilde{a}\left(0, x, \tau, t^{l_{*}} \xi\right) .
$$

The symbol $\tilde{a}(t, x, \tau, \tilde{\xi})$ resulting from (4.19), with $a(t, x, \tau, \xi)$ being $\sigma_{\psi}^{m}(a)(t, x, \tau, \xi)$ for some $a \in S_{\mathrm{tr}, \mathrm{cl}}^{m ;}\left([0, T) \times \Omega \times \mathbb{R}^{1+n}\right)$, shall be denoted by $\tilde{\sigma}_{\psi}^{m ; \lambda}(a)(t, x, \tau, \tilde{\xi})$. 
Proposition 4.21. The following short sequence is split exact:

$$
\begin{aligned}
& 0 \longrightarrow S_{\mathrm{tr}, \mathrm{cl}}^{m-1 ; \lambda}\left([0, T) \times \Omega \times \mathbb{R}^{1+n}\right) \longrightarrow S_{\mathrm{tr}, \mathrm{cl}}^{m ; \lambda}\left([0, T) \times \Omega \times \mathbb{R}^{1+n}\right) \\
& \stackrel{\sigma^{m ; \lambda}}{\longrightarrow} \Sigma S_{\mathrm{tr}, \mathrm{cl}}^{m ; \lambda}\left([0, T) \times \Omega \times \mathbb{R}^{1+n}\right) \longrightarrow 0,
\end{aligned}
$$

where $\sigma^{m ; \lambda}=\left(\sigma_{\psi}^{m}, \sigma_{b}^{m ; \lambda}\right)$ is the principal symbol map.

REMARK 4.22. Assume that $a \in S_{\mathrm{tr}, \mathrm{cl}}^{m ; \lambda}\left(\overline{\mathbb{R}}_{+} \times \Omega \times \mathbb{R}^{1+n}\right)$ satisfies the symbol estimates globally in $t \in \overline{\mathbb{R}}_{+}$, i.e., $T=\infty$ and the constants $C_{j k \alpha \beta T^{\prime} K}>0$ in (4.1) can be chosen independently of $T^{\prime}>0$. Then, for all $\alpha, \beta \in \mathbb{N}^{n}, K \Subset \Omega$, there is a constant $C_{\alpha \beta K}>0$ (also depending on $s, \delta \in \mathbb{R}$ ) such that

$$
\begin{array}{r}
\left\|\kappa_{\langle\xi\rangle^{-1}}^{(\delta+m)} \partial_{x}^{\beta} \partial_{\xi}^{\alpha} \operatorname{op}_{t}^{+}(a)(x, \xi) \kappa_{\langle\xi\rangle}^{(\delta)}\right\|_{\mathcal{L}\left(H^{s, \delta ; \lambda}\left(\mathbb{R}_{+}\right), H^{s-m, \delta+m ; \lambda}\left(\mathbb{R}_{+}\right)\right)} \leq C_{\alpha \beta K}\langle\xi\rangle^{m-|\alpha|} \\
(x, \xi) \in K \times \mathbb{R}^{n}
\end{array}
$$

This shows that the function

$$
\Omega \times \mathbb{R}^{n} \rightarrow \mathcal{L}\left(H^{s, \delta ; \lambda}\left(\mathbb{R}_{+}\right), H^{s-m, \delta+m ; \lambda}\left(\mathbb{R}_{+}\right)\right), \quad(x, \xi) \mapsto \mathrm{op}_{t}^{+}(a)(x, \xi),
$$

is an operator-valued symbol with involved group actions in the sense of Schulze, see Schulze [20, Section 1.3]. Note, however, that in improving the order of the symbol from $m$ to $m-1$ (e.g., by subtracting off a leading homogeneous component), one of the groups is altered, see also Proposition 4.25, Remark 4.26.

To prove (4.20), it suffices to verify that the set

$$
\left\{\langle\xi\rangle^{-\beta_{*} m+|\alpha|} \partial_{x}^{\beta} \partial_{\xi}^{\alpha} a\left(\langle\xi\rangle^{-\beta_{*}} t, x,\langle\xi\rangle^{\beta_{*}} \tau, \xi\right) ;(x, \xi) \in K \times \mathbb{R}^{n}\right\} \subset S_{\mathrm{tr}}^{m ; \lambda}\left(\overline{\mathbb{R}}_{+} \times \mathbb{R}\right)
$$

is bounded for any $K \Subset \Omega$, because of

$$
\kappa_{\langle\xi\rangle-1}^{(\delta+m)} \partial_{x}^{\beta} \partial_{\xi}^{\alpha} \mathrm{op}_{t}^{+}(a)(x, \xi) \kappa_{\langle\xi\rangle}^{(\delta)}=\langle\xi\rangle^{\beta_{*} m l_{*}} \mathrm{op}_{t}^{+}\left(\partial_{x}^{\beta} \partial_{\xi}^{\alpha} a\left(\langle\xi\rangle^{-\beta_{*}} t, x,\langle\xi\rangle^{\beta_{*}} \tau, \xi\right)\right) .
$$

Indeed, for $(t, x, \tau, \xi) \in \overline{\mathbb{R}}_{+} \times K \times \mathbb{R}^{1+n}$,

$$
\begin{aligned}
& \left|\partial_{t}^{k} \partial_{\tau}^{j}\left(\langle\xi\rangle^{-m+|\alpha|}\langle\xi\rangle^{\beta_{*} m l_{*}} \partial_{x}^{\beta} \partial_{\xi}^{\alpha} a\left(\langle\xi\rangle^{-\beta_{*}} t, x,\langle\xi\rangle^{\beta_{*}} \tau, \xi\right)\right)\right| \\
& \quad \leq C\langle\xi\rangle^{-\beta_{*} m+|\alpha|+\beta_{*}(j-k)} \Phi\left(\langle\xi\rangle^{-\beta_{*}} t,\langle\xi\rangle^{\beta_{*}} \tau, \xi\right)^{m-j}\left(\langle\xi\rangle^{-\beta_{*}}+\langle\xi\rangle^{-\beta_{*}} t\right)^{-k}\langle\xi\rangle^{-|\alpha|} \\
& \leq C^{\prime}\left(1+|\tau|+t^{l_{*}}\right)^{m-j}(1+t)^{-k}
\end{aligned}
$$

for some constants $C, C^{\prime}>0$. This inequality immediately yields (4.21).

Proposition 4.23. The symbol a arising from the operator $A$ in (1.1), i.e.,

$$
a(t, x, \tau, \xi)=\sum_{j+|\alpha| \leq m} a_{j \alpha}(t, x) t^{\left(j+\left(l_{*}+1\right)|\alpha|-m\right)^{+}} \tau^{j} \xi^{\alpha},
$$

belongs to the space $S_{\mathrm{tr}, \mathrm{cl}}^{m ; \lambda}\left([0, T) \times \Omega \times \mathbb{R}^{1+n}\right)$. Furthermore, the principal symbols $\sigma_{\psi}^{m}(a)$, $\sigma_{b}^{m ; \lambda}(a)$ are identified in (1.2) and (1.3), respectively.

Proof. Upon setting $d(t, x, \tau, \xi):=a_{j \alpha}(t, x) t^{\left(j+\left(l_{*}+1\right)|\alpha|-m\right)^{+}} \tau^{j} \xi^{\alpha}$ for $j+|\alpha| \leq m$, it suffices to prove that

$$
d(t, x, \tau, \xi) \in S_{\mathrm{cl}}^{j+|\alpha|, \bar{m} ; \lambda}\left([0, T) \times \Omega \times \mathbb{R}^{1+n}\right) \subseteq S_{\mathrm{cl}}^{m, m ; \lambda}\left([0, T) \times \Omega \times \mathbb{R}^{1+n}\right),
$$


where $\bar{m}=\min \left\{m, j+\left(l_{*}+1\right)|\alpha|\right\}$,

$$
\begin{aligned}
\sigma_{\psi}^{j+|\alpha|}(d)(t, x, \tau, \xi) & =d(t, x, \tau, \xi), \\
\sigma_{b}^{\bar{m}-h ; \lambda}(d)(t, x, \tau, \xi) & =\frac{\partial^{h} a_{j \alpha}}{\partial t^{h}}(0, x) t^{j+\left(l_{*}+1\right)|\alpha|-\bar{m}+h} \tau^{j} \xi^{\alpha}, \quad h=0,1,2, \ldots,
\end{aligned}
$$

(all other symbols vanish), and, in addition, $d$ satisfies the $\lambda$-transmission property.

Obviously, $a_{j \alpha}(t, x) \in S_{\mathrm{tr}, \mathrm{cl}}^{0 ; \lambda}\left([0, T) \times \Omega \times \mathbb{R}^{1+n}\right)$, where $\sigma_{\psi}^{0}\left(a_{j \alpha}\right)(t, x, \tau, \xi)=a_{j \alpha}(t, x)$, $\sigma_{b}^{-h ; \lambda}\left(a_{j \alpha}\right)(t, x, \tau, \xi)=\frac{\partial^{h} a_{j \alpha}}{\partial t^{h}}(0, x) t^{h}$ for $h=0,1,2, \ldots$ Therefore, in (4.22), we may assume that $a_{j \alpha}(t, x)=1$ for all $(t, x) \in[0, T) \times \Omega$.

Then we have

$$
\begin{aligned}
\partial_{t}^{k} \partial_{x}^{\beta} \partial_{\tau}^{j^{\prime}} \partial_{\xi}^{\alpha^{\prime}} d(t, x, \tau, \xi) \\
\quad= \begin{cases}C t^{j+\left(l_{*}+1\right)|\alpha|-\bar{m}-k} \tau^{j-j^{\prime}} \xi^{\alpha-\alpha^{\prime}} & \text { if } k \leq j+\left(l_{*}+1\right)|\alpha|-\bar{m}, j^{\prime} \leq j, \alpha^{\prime} \leq \alpha, \\
0 & \text { otherwise }\end{cases}
\end{aligned}
$$

for a certain constant $C=C_{j j^{\prime} k \alpha \alpha^{\prime} \beta \beta^{\prime}}$. In the first case, we get

$$
\begin{array}{r}
\left|\partial_{t}^{k} \partial_{x}^{\beta} \partial_{\tau}^{j^{\prime}} \partial_{\xi}^{\alpha^{\prime}} d(t, x, \tau, \xi)\right| \leq C\left(\langle\xi\rangle^{-\beta_{*}}+t\right)^{j+|\alpha|-\bar{m}-k}|\tau|^{j-j^{\prime}}\left(\left(\langle\xi\rangle^{-\beta_{*}}+t\right)^{l_{*}}\langle\xi\rangle\right)^{|\alpha|}\langle\xi\rangle^{-\left|\alpha^{\prime}\right|} \\
\leq C \Phi^{j+|\alpha|-j^{\prime}}\left(\langle\xi\rangle^{-\beta_{*}}+t\right)^{j+|\alpha|-\bar{m}-k}\langle\xi\rangle^{-\left|\alpha^{\prime}\right|}
\end{array}
$$

showing that $d(t, x, \tau, \xi) \in S^{j+|\alpha|, \bar{m} ; \lambda}\left([0, T) \times \Omega \times \mathbb{R}^{1+n}\right)$. Also

$$
d(t, x, \tau, \xi) \in S_{\mathrm{cl}}^{j+|\alpha|, \bar{m} ; \lambda}\left([0, T) \times \Omega \times \mathbb{R}^{1+n}\right)
$$

with $\sigma_{\psi}^{j+|\alpha|}(d)=d, \sigma_{b}^{\bar{m}}(d)=d$ is now obvious.

It remains to prove that $d$ satisfies the $\lambda$-transmission property. But

$$
\partial_{t}^{k} d\left(0, x,\langle\xi\rangle^{\beta_{*}} \tau, \xi\right)= \begin{cases}C\left(\langle\xi\rangle^{\beta_{*}} \tau\right)^{j} \xi^{\alpha} & \text { if } k=j+\left(l_{*}+1\right)|\alpha|-\bar{m} \\ 0 & \text { otherwise }\end{cases}
$$

and, in the first case, we get

$$
\partial_{t}^{k} d\left(0, x,\langle\xi\rangle^{\beta_{*}} \tau, \xi\right)=\tau^{j}\langle\xi\rangle^{\beta_{*}(\bar{m}+k)-|\alpha|} \xi^{\alpha} \in S^{\beta_{*}(\bar{m}+k)}\left(\Omega \times \mathbb{R}^{n} ; H_{j+1}\right)
$$

as required.

4.3. Singular Green, trace, and potential symbols. Here, we follow the presentation in Schulze [20, Section 4.2.3], while adopting several obvious changes. Some of these are required due to the fact that different group actions are involved, cf. Proposition 4.25, Remark 4.26 .

Definition 4.24. For $m \in \mathbb{Z}, d, N_{-}, N_{+} \in \mathbb{N}$, the class $R_{G}^{m, d ; \lambda}\left(\Omega \times \mathbb{R}^{n} ; N_{-}, N_{+}\right)$ consists of all operator-valued symbols

$$
\mathfrak{g}(x, \xi)=\left(\begin{array}{lll}
g(x, \xi) & k(x, \xi) \\
s(x, \xi) & q(x, \xi)
\end{array}\right): \begin{array}{ccc}
\mathcal{S}\left(\overline{\mathbb{R}}_{+}\right) & & \mathcal{S}\left(\overline{\mathbb{R}}_{+}\right) \\
\mathbb{C}^{N_{-}} & & \mathbb{C}^{N_{+}}
\end{array}
$$


for $(x, \xi) \in \Omega \times \mathbb{R}^{n}$, where

$$
g(x, \xi) u(t)=\sum_{k=0}^{d} \int_{0}^{\infty} g_{k}\left(x, \xi,\langle\xi\rangle^{\beta_{*}} t,\langle\xi\rangle^{\beta_{*}} t^{\prime}\right) \partial_{t^{\prime}}^{k} u\left(t^{\prime}\right) d t^{\prime}
$$

for suitable $g_{k} \in S_{\mathrm{cl}, \beta_{*}}^{\beta_{*}(m-k+1)}\left(\Omega \times \mathbb{R}^{n} ; \mathcal{S}\left(\overline{\mathbb{R}}_{+} \times \overline{\mathbb{R}}_{+}\right)\right), k=0,1, \ldots, d$; further, $s(x, \xi)=$ $\left(s_{1}(x, \xi), \ldots, s_{N_{+}}(x, \xi)\right)$,

$$
s_{j}(x, \xi) u(t)=\sum_{k=0}^{d} \int_{0}^{\infty} s_{j k}\left(x, \xi,\langle\xi\rangle^{\beta_{*}} t^{\prime}\right) \partial_{t^{\prime}}^{k} u\left(t^{\prime}\right) d t^{\prime}
$$

for suitable $s_{j k} \in S_{\mathrm{cl}, \beta_{*}}^{\beta_{*}(m-k+1 / 2)}\left(\Omega \times \mathbb{R}^{n} ; \mathcal{S}\left(\overline{\mathbb{R}}_{+}\right)\right), j=1, \ldots, N_{+}, k=0,1, \ldots, d$,

$$
k(x, \xi) c=\sum_{l=1}^{N_{-}} c_{l} k_{l}\left(x, \xi,\langle\xi\rangle^{\beta_{*}} t\right), \quad c=\left(c_{1}, \ldots, c_{N_{-}}\right) \in \mathbb{C}^{N_{-}}
$$

for suitable $k_{l} \in S_{\mathrm{cl}, \beta_{*}}^{\beta_{*}^{(m+1 / 2)}}\left(\Omega \times \mathbb{R}^{n} ; \mathcal{S}\left(\overline{\mathbb{R}}_{+}\right)\right)$, and $q(x, \xi) \in S_{\mathrm{cl}, \beta_{*}}^{\beta_{*} m}\left(\Omega \times \mathbb{R}^{n} ; \mathbb{C}^{N_{-}}, \mathbb{C}^{N_{+}}\right)$is a symbol of order $\beta_{*} m$ with values in $N_{+} \times N_{-}$-matrices.

In case $N_{-}=N_{+}=0$, we also write $R_{G}^{m, d ; \lambda}\left(\Omega \times \mathbb{R}^{n}\right):=R_{G}^{m, d ; \lambda}\left(\Omega \times \mathbb{R}^{n} ; 0,0\right)$.

In Definition 4.24, $g \in R_{G}^{m, d ; \lambda}\left(\Omega \times \mathbb{R}^{n}\right)$ is called a singular Green symbol of order $m$ and type $d, t$ is called a trace symbol of order $m$ and type $d$, and $k$ is called a potential symbol of order $m$ (always with degeneracy of type $\lambda$ ).

Note that the symbol $\mathfrak{g}$ in (4.23) belongs to $C^{\infty}\left(\Omega \times \mathbb{R}^{n} ; B^{-\infty, d}\left(\mathbb{R}_{+} ; N_{-}, N_{+}\right)\right)$. Moreover, the limit is seen

$$
\left(\begin{array}{cc}
\bar{g}(x, \xi) & \bar{k}(x, \xi) \\
\bar{s}(x, \xi) & \bar{q}(x, \xi)
\end{array}\right):=\lim _{\nu \rightarrow \infty} \nu^{-m}\left(\begin{array}{cc}
\kappa_{\nu}^{(\delta+m)} & 0 \\
0 & 1_{\mathbb{C}^{N_{+}}}
\end{array}\right)^{-1} \mathfrak{g}(x, \xi)\left(\begin{array}{cc}
\kappa_{\nu}^{(\delta)} & 0 \\
0 & 1_{\mathbb{C}^{N_{-}}}
\end{array}\right)
$$

to exist (in an appropriate topology) for all $(x, \xi) \in \Omega \times\left(\mathbb{R}^{n} \backslash 0\right)$, and any $\delta \in \mathbb{R}$, where

$$
\begin{gathered}
\bar{g}(x, \xi) u(t)=\sum_{k=0}^{d} \int_{0}^{\infty}|\xi|^{\beta_{*}(m+1)} \sigma_{\psi}^{\beta_{*}(m-k+1)}\left(g_{k}\right)\left(x, \frac{\xi}{|\xi|},|\xi|^{\beta_{*}} t,|\xi|^{\beta_{*}} t^{\prime}\right) \partial_{t^{\prime}}^{k} u\left(t^{\prime}\right) d t^{\prime} \\
\bar{s}_{j}(x, \xi) u(t)=\sum_{k=0}^{d} \int_{0}^{\infty}|\xi|^{\beta_{*}(m+1 / 2)} \sigma_{\psi}^{\beta_{*}(m-k+1 / 2)}\left(t_{j k}\right)\left(x, \frac{\xi}{|\xi|},|\xi|^{\beta_{*}} t^{\prime}\right) \partial_{t^{\prime}}^{k} u\left(t^{\prime}\right) d t^{\prime} \\
\bar{k}(x, \xi) c=\sum_{l=1}^{N_{-}} c_{l}|\xi|^{\beta_{*}(m+1 / 2)} \sigma_{\psi}^{\beta_{*}(m+1 / 2)}\left(k_{l}\right)\left(x, \frac{\xi}{|\xi|},|\xi|^{\beta_{*}} t\right), \\
\bar{q}(x, \xi)=|\xi|^{\beta_{*} m} \sigma_{\psi}^{\beta_{*} m}(q)\left(x, \frac{\xi}{|\xi|}\right) .
\end{gathered}
$$

In fact, we have 
Proposition 4.25. Let $\mathfrak{g}_{j} \in C^{\infty}\left(\Omega \times \mathbb{R}^{n} ; B^{-\infty, d}\left(\mathbb{R}_{+} ; N_{-}, N_{+}\right)\right)$for $j=0,1,2 \ldots$ satisfy

$$
\mathfrak{g}_{j}(x, \nu \xi)=\nu^{m-j}\left(\begin{array}{cc}
\kappa_{\nu}^{(\delta+m-j)} & 0 \\
0 & 1_{\mathbb{C}^{N_{+}}}
\end{array}\right) \mathfrak{g}_{j}(x, \xi)\left(\begin{array}{cc}
\kappa_{\nu}^{(\delta)} & 0 \\
0 & 1_{\mathbb{C}^{N_{-}}}
\end{array}\right)^{-1}, \quad \nu>0
$$

for all $(x, \xi) \in \Omega \times\left(\mathbb{R}^{n} \backslash 0\right)$ and any $\delta \in \mathbb{R}$. Then there is a symbol $\mathfrak{g} \in R_{G}^{m, d ; \lambda}\left(\Omega \times \mathbb{R}^{n}\right.$; $\left.N_{-}, N_{+}\right)$, such that, for all $J \in \mathbb{N}$,

$$
\mathfrak{g}-\sum_{j<J} \chi(|\xi|) \mathfrak{g}_{j} \in R_{G}^{m-j, d ; \lambda}\left(\Omega \times \mathbb{R}^{n} ; N_{-}, N_{+}\right) .
$$

Conversely, for each $\mathfrak{g} \in R_{G}^{m, d ; \lambda}\left(\Omega \times \mathbb{R}^{n} ; N_{-}, N_{+}\right)$, there are (uniquely determined) symbols $\mathfrak{g}_{j} \in C^{\infty}\left(\Omega \times\left(\mathbb{R}^{n} \backslash 0\right) ; B^{-\infty, d}\left(\mathbb{R}_{+} ; N_{-}, N_{+}\right)\right)$for $j=0,1,2, \ldots$ satisfying (4.25) such that (4.26) holds.

We write

$$
\sigma_{b}^{m-j ; \lambda}(\mathfrak{g})(x, \xi):=\mathfrak{g}_{j}(x, \xi), \quad j=0,1,2, \ldots,
$$

Note that $\mathfrak{g}_{0}$ has already been calculated in (4.24).

REMARK 4.26. When going over from (4.24) to (4.25), one of the groups involved is altered from $\left\{\kappa_{\nu}^{(\delta+m)}\right\}_{\nu>0}$ to $\left\{\kappa_{\nu}^{(\delta+m-j)}\right\}_{\nu>0}$.

From the similar situation of $l_{*}=0$, it is known that the crucial step for establishing compositions within in the calculus is to prove of the next result:

LemMA 4.27. Let $a \in S_{\mathrm{tr}, \mathrm{cl}}^{m ; \lambda}\left(\overline{\mathbb{R}}_{+} \times \Omega \times \mathbb{R}^{1+n}\right), b \in S_{\mathrm{tr}, \mathrm{cl}}^{m^{\prime} ; \lambda}\left(\overline{\mathbb{R}}_{+} \times \Omega \times \mathbb{R}^{1+n}\right)$ be such that, for $t \geq 1, a(t, x, \tau, \xi)=t^{-m} \tilde{a}\left(x, t \tau, t^{l_{*}+1} \xi\right), b(t, x, \tau, \xi)=t^{-m^{\prime}} \tilde{b}\left(x, t \tau, t^{l_{*}+1} \xi\right)$ for some $\tilde{a} \in S_{\mathrm{cl}}^{m}\left(\Omega \times \mathbb{R}^{1+n}\right), \tilde{b} \in S_{\mathrm{cl}}^{m^{\prime}}\left(\Omega \times \mathbb{R}^{1+n}\right)$. (For this assumption, see Lemma 4.13 , at least as concerns the leading homogeneous component.) Then

$$
\mathrm{op}^{+}(a \circ b)-\mathrm{op}^{+}(a) \mathrm{op}^{+}(b) \in R_{G}^{m+m^{\prime},\left(m^{\prime}\right)^{+} ; \lambda}\left(\Omega \times \mathbb{R}^{n}\right),
$$

where $\circ$ denotes the Leibniz product with respect to $t$, cf. (3.2).

Proof. For a proof in case $l_{*}=0$, see Schrohe-Schulze [19, Proposition 5.2.1]. Here, we only sketch the proof.

We may assume that $a=0, b=0$ when $t \geq 1$. Further we may assume that $\partial_{t}^{k} a\left(0, x,\langle\xi\rangle^{\beta_{*}} \tau, \xi\right) \in S^{\beta_{*}(m+k)}\left(\Omega \times \mathbb{R}^{n} ; H_{0}\right)$ for $k=0,1,2, \ldots$, and similarly for $b$, because of contributions coming from polynomial components are easily dealt with. The operator in (4.27) equals

$$
\left(r^{+} \text {op }(a) e^{-} \varepsilon^{*}\right)\left(\varepsilon^{*} r^{-} \operatorname{op}(b) e^{+}\right),
$$

where $e^{-}, r^{-}$are defined by analogy to $e^{+}, r^{+}$, but with $\mathbb{R}_{-}$in place of $\mathbb{R}_{+}$, and $\varepsilon^{*}$ is the reflection operator, $\varepsilon^{*} v(t):=v(-t)$ for functions $v$ on $\mathbb{R}_{+}$and $\mathbb{R}_{-}$, respectively. Hence, it suffices to prove that

$$
r^{+} \operatorname{op}(a) e^{-} \varepsilon^{*} \in R_{G}^{m, 0 ; \lambda}\left(\Omega \times \mathbb{R}^{n}\right), \quad \varepsilon^{*} r^{-} \operatorname{op}(b) e^{+} \in R_{G}^{m^{\prime}, 0 ; \lambda}\left(\Omega \times \mathbb{R}^{n}\right) .
$$


We treat the first operator only; the treatment the second one is completely analogous. A brief calculations gives that

$$
r^{+} \operatorname{op}(a) e^{-} \varepsilon^{*} u(t)=\int_{0}^{\infty} g\left(x, \xi,\langle\xi\rangle^{\beta_{*}} t,\langle\xi\rangle^{\beta_{*}} t^{\prime}\right) u\left(t^{\prime}\right) d t^{\prime},
$$

where $g\left(x, \xi, t, t^{\prime}\right)=\tilde{a}\left(\langle\xi\rangle^{-\beta_{*}} t, x,\langle\xi\rangle^{-\beta_{*}}\left(t+t^{\prime}\right), \xi\right), \tilde{a}(t, x, s, \xi)=F_{\tau \rightarrow s}^{-1}\{a(t, x, \tau, \xi)\}$. We get $\partial_{t}^{k} \tilde{a}\left(0, x,\langle\xi\rangle^{-\beta_{*}} s, \tau\right) \in S^{\beta_{*}(m+k+1)}\left(\Omega \times \mathbb{R}^{n} ; \mathcal{S}\left(\overline{\mathbb{R}}_{+}\right)\right)$for $k=0,1,2, \ldots$, due to the characterization of the space $H_{0}$ as the Fourier image of $\mathcal{S}\left(\overline{\mathbb{R}}_{+}\right) \oplus \mathcal{S}\left(\bar{R}_{-}\right)$. Consequently,

$$
g\left(x, \xi, t, t^{\prime}\right) \sim \sum_{k=0}^{\infty} \frac{t^{k}}{k !}\langle\xi\rangle^{-\beta_{*} k} \partial_{t}^{k} \tilde{a}\left(0, x,\langle\xi\rangle^{-\beta_{*}}\left(t+t^{\prime}\right), \tau\right),
$$

with each summand on the right-hand side belonging to $S^{\beta_{*}(m+1)}\left(\Omega \times \mathbb{R}^{n} ; \mathcal{S}\left(\overline{\mathbb{R}}_{+} \times \overline{\mathbb{R}}_{+}\right)\right)$, but with slight improvement caused by multiplying with powers of $t$ (each multiplication by $t$ produces an increment of $1 / l_{*}$ of the parameter $\delta$ appearing, in (4.25), in the group on the left). This yields estimates for the kernel function $g$ in the space $S^{\beta_{*}(m+1)}\left(\Omega \times \mathbb{R}^{n}\right.$; $\left.\mathcal{S}\left(\overline{\mathbb{R}}_{+} \times \overline{\mathbb{R}}_{+}\right)\right)$

These estimates are then improved to estimates for $g$ in the space $S_{\mathrm{cl}, \beta_{*}}^{\beta_{*}(m+1)}\left(\Omega \times \mathbb{R}^{n}\right.$; $\left.\mathcal{S}\left(\overline{\mathbb{R}}_{+} \times \overline{\mathbb{R}}_{+}\right)\right)$by subtracting off leading homogeneous terms. For instance, if one has $a\left(\nu^{-1} t, x, \nu \tau, \nu^{l_{*}+1} \xi\right)=\nu^{m} a(t, x, \tau, \xi)$ for $\nu>0$, then

$$
\tilde{a}\left(\nu^{-1} t, x, \nu^{-1} s, \nu^{l_{*}+1} \xi\right)=\nu^{m+1} \tilde{a}(t, x, s, \xi), \quad \nu>0,
$$

and, therefore,

$$
g\left(t, t^{\prime}, x, \xi\right)=\langle\xi\rangle^{\beta_{*}(m+1)} \tilde{a}\left(t, x, t+t^{\prime}, \frac{\xi}{\langle\xi\rangle}\right),
$$

where the remaining term is seen to belong to $S^{\beta_{*} m}\left(\Omega \times \mathbb{R}^{n} ; \mathcal{S}\left(\overline{\mathbb{R}}_{+} \times \overline{\mathbb{R}}_{+}\right)\right)$by the previous considerations.

In this way, the proof is finally completed.

4.4. Boundary symbols. Having constructed interior symbols in Section 4.1 and singular Green, trace, and potential symbols in Section 4.2, respectively, we are now able to provide the calculus of complete boundary symbols. These are operator-valued symbols depending on $(x, \xi) \in \Omega \times \mathbb{R}^{n}$ and taking values in the calculus $B^{m, d ; \lambda}\left(\mathbb{R}_{+} ; N_{-}, N_{+}\right)$on the half-axis.

Definition 4.28. For $m \in \mathbb{Z}, d, N_{-}, N_{+} \in \mathbb{N}$, the space $R^{m, d ; \lambda}\left(\Omega \times \mathbb{R}^{n} ; N_{-}, N_{+}\right)$ consists of all operator-valued symbols

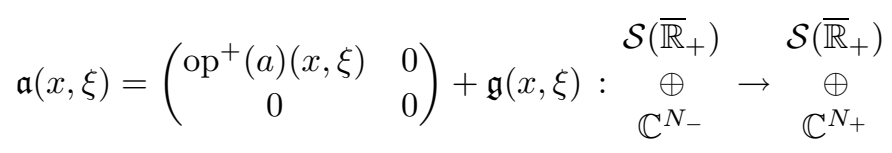

for $(x, \xi) \in \Omega \times \mathbb{R}^{n}$, where $a \in S_{\mathrm{tr}, \mathrm{cl}}^{m ; \lambda}\left(\overline{\mathbb{R}}_{+} \times \Omega \times \mathbb{R}^{1+n}\right)$ that, for $t \geq 1$, is of the form $t^{-m} \tilde{a}\left(x, t \tau, t^{l_{*}+1} \xi\right)$ for some $\tilde{a} \in S_{\mathrm{cl}}^{m}\left(\Omega \times \mathbb{R}^{1+n}\right)$ and $\mathfrak{g} \in R_{G}^{m, d ; \lambda}\left(\Omega \times \mathbb{R}^{n} ; N_{-}, N_{+}\right)$.

The symbol $\mathfrak{a}=\mathfrak{a}(x, \xi)$ in $(4.28)$ is called a complete boundary symbol of order $m$ and type $d$ (with degeneracy of type $\lambda$ ). 
We set

$$
\sigma_{b}^{m-j ; \lambda}(\mathfrak{a})(x, \xi):=\left(\begin{array}{cc}
\mathrm{op}^{+}\left(\sigma_{b}^{m-j ; \lambda}(a)\right)(x, \xi) & 0 \\
0 & 0
\end{array}\right)+\sigma_{b}^{m-j ; \lambda}(\mathfrak{g})(x, \xi),
$$

$j=0,1,2, \ldots$ Note that $\sigma_{b}^{m-j ; \lambda}(\mathfrak{a}) \in C^{\infty}\left(\Omega \times\left(\mathbb{R}^{n} \backslash 0\right) ; B^{m-j, d ; \lambda}\left(\mathbb{R}_{+} ; N_{-}, N_{+}\right)\right)$and

$$
\sigma_{b}^{m-j ; \lambda}(\mathfrak{a})(x, \nu \xi)=\nu^{m-j}\left(\begin{array}{cc}
\kappa_{\nu}^{(\delta+m-j)} & 0 \\
0 & 1_{\mathbb{C}^{N_{+}}}
\end{array}\right) \sigma_{b}^{m-j ; \lambda}(\mathfrak{a})(x, \xi)\left(\begin{array}{cc}
\kappa_{\nu}^{(\delta)} & 0 \\
0 & 1_{\mathbb{C}^{N_{-}}}
\end{array}\right)^{-1}
$$

for $\nu>0, j=0,1,2, \ldots$

Proposition 4.29. Let $\mathfrak{a}_{j} \in R^{m-j, d ; \lambda}\left(\Omega \times \mathbb{R}^{n} ; N_{-}, N_{+}\right)$for $j=0,1,2, \ldots$ Then there is a symbol $\mathfrak{a} \in R^{m, d ; \lambda}\left(\Omega \times \mathbb{R}^{n} ; N_{-}, N_{+}\right)$such that, for all $J \in \mathbb{N}$,

$$
\mathfrak{a}-\sum_{j<J} \mathfrak{a}_{j} \in R^{m-J, d ; \lambda}\left(\Omega \times \mathbb{R}^{n} ; N_{-}, N_{+}\right) .
$$

In case (4.29) holds, we write

$$
\mathfrak{a} \sim \sum_{j=0}^{\infty} \mathfrak{a}_{j}
$$

Proposition 4.30. For the symbol classes $R^{m, d ; \lambda}\left(\Omega \times \mathbb{R}^{n} ; N_{-}, N_{+}\right)$for $m \in \mathbb{Z}$ the following rules hold:

(a) $\partial_{x}^{\beta} \partial_{\xi}^{\alpha} \mathfrak{a} \in R^{m-|\alpha| ; \lambda}\left(\Omega \times \mathbb{R}^{n} ; N_{-}, N_{+}\right)$if $\mathfrak{a} \in R^{m ; \lambda}\left(\Omega \times \mathbb{R}^{n} ; N_{-}, N_{+}\right)$.

(b) $\mathfrak{a} \mathfrak{b} \in R^{m+m^{\prime} ; \lambda}\left(\Omega \times \mathbb{R}^{n} ; N_{-}, N_{+}\right)$if $\mathfrak{a} \in R^{m ; \lambda}\left(\Omega \times \mathbb{R}^{n} ; \tilde{N}, N_{+}\right), \mathfrak{b} \in R^{m^{\prime} ; \lambda}\left(\Omega \times \mathbb{R}^{n}\right.$; $\left.N_{-}, \tilde{N}\right)$. Furthermore,

$$
\sigma_{b}^{m+m^{\prime} ; \lambda}(\mathfrak{a} \mathfrak{b})=\sigma_{b}^{m ; \lambda}(\mathfrak{a}) \sigma_{b}^{m^{\prime} ; \lambda}(\mathfrak{b}) .
$$

(c) $\mathfrak{a} \circ \mathfrak{b} \in R^{m+m^{\prime} ; \lambda}\left(\Omega \times \mathbb{R}^{n} ; N_{-}, N_{+}\right)$if $\mathfrak{a} \in R^{m, \lambda}\left(\Omega \times \mathbb{R}^{n} ; \tilde{N}, N_{+}\right), \mathfrak{b} \in R^{m^{\prime} ; \lambda}\left(\Omega \times \mathbb{R}^{n} ;\right.$ $\left.N_{-}, \tilde{N}\right)$, where $\circ$ is the Leibniz product. Furthermore,

$$
(\mathfrak{a} \circ \mathfrak{b})(x, \xi) \sim \sum_{j, \alpha} \frac{1}{\alpha !} \partial_{\xi}^{\alpha} \mathfrak{a}(x, \xi) D_{x}^{\alpha} \mathfrak{b}(x, \xi) .
$$

Definition 4.31. The principal symbol space $\Sigma R^{m, d ; \lambda}\left(\Omega \times \mathbb{R}^{n} ; N_{-}, N_{+}\right)$for the symbol class $R^{m, d ; \lambda}\left(\Omega \times \mathbb{R}^{n} ; N_{-}, N_{+}\right)$consists of all couples $(a, \mathfrak{a})$, where $a \in S_{\mathrm{tr}}^{(m)}\left(\overline{\mathbb{R}}_{+} \times \Omega \times\right.$ $\left.\left(\mathbb{R}^{1+n} \backslash 0\right)\right)$ is such that

$$
a(t, x, \tau, \xi)=\tilde{a}\left(t, x, \tau, t^{l_{*}} \tau\right)
$$

for a certain $\tilde{a} \in S^{(m)}\left(\overline{\mathbb{R}}_{+} \times \Omega \times\left(\mathbb{R}^{1+n} \backslash 0\right)\right), \tilde{a}=\tilde{a}(t, x, \tau, \tilde{\xi})$ is independent of $t$ for $t \geq 1$, and $\mathfrak{a} \in C^{\infty}\left(\Omega \times\left(\mathbb{R}^{n} \backslash 0\right) ; B^{m, d ; \lambda}\left(\mathbb{R}_{+} ; N_{-}, N_{+}\right)\right)$satisfies

$$
\mathfrak{a}(x, \nu \xi)=\nu^{m}\left(\begin{array}{cc}
\kappa_{\nu}^{(\delta+m)} & 0 \\
0 & 1_{\mathbb{C}^{N_{+}}}
\end{array}\right) \mathfrak{a}(x, \xi)\left(\begin{array}{cc}
\kappa_{\nu}^{(\delta)} & 0 \\
0 & 1_{\mathbb{C}^{N_{-}}}
\end{array}\right)^{-1}, \quad \nu>0,
$$

for any $\delta \in \mathbb{R}$. Moreover, it is required that

$$
\sigma_{\psi}^{m}(\mathfrak{a})(t, \tau ; x, \xi)=\tilde{a}\left(0, x, \tau, t^{l_{*}} \xi\right) .
$$


Proposition 4.32. The following short sequence is split exact:

$$
\begin{aligned}
0 \longrightarrow B^{m-1, d ; \lambda}\left(\Omega \times \mathbb{R}^{n} ; N_{-}, N_{+}\right) \longrightarrow & B^{m, d ; \lambda}\left(\Omega \times \mathbb{R}^{n} ; N_{-}, N_{+}\right) \\
& \stackrel{\sigma^{m ; \lambda}}{\longrightarrow} \Sigma R^{m, d ; \lambda}\left(\Omega \times \mathbb{R}^{n} ; N_{-}, N_{+}\right) \longrightarrow 0 .
\end{aligned}
$$

Here, $\sigma^{m ; \lambda}=\left(\sigma_{\psi}^{m}, \sigma_{b}^{m ; \lambda}\right)$ is the principal symbol map.

5. The local calculus. Now, we present the local calculus on $(0, T) \times \Omega$. Again, we follow the exposition in Schulze [20, Section 4.3], where, this time, no further changes are necessary. For this reason, proofs are nearly completely dropped. They can simply be added by the reader following the lines of the quoted reference.

Definition 5.1. Let $m \in \mathbb{Z}, d \in \mathbb{N}$.

(a) The class $B^{-\infty, d ; \lambda}\left((0, T) \times \Omega ; N_{-}, N_{+}\right)$consists of all operators

$$
\mathfrak{G}=\left(\begin{array}{ll}
G & K \\
S & Q
\end{array}\right): \begin{array}{ccc}
C_{\mathrm{comp}}^{\infty}([0, T) \times \Omega) & \oplus & C^{\infty}([0, T) \times \Omega) \\
& C_{\mathrm{comp}}^{\infty}\left(\Omega ; \mathbb{C}^{N_{-}}\right)
\end{array} \quad \begin{gathered}
\oplus \\
C^{\infty}\left(\Omega ; \mathbb{C}^{N_{+}}\right)
\end{gathered}
$$

where

$$
\begin{aligned}
G u(t, x) & =\sum_{k=0}^{d} \int_{0}^{T} \int_{\Omega} g_{k}\left(t, x, t^{\prime}, x^{\prime}\right) \partial_{t^{\prime}}^{k} u\left(t^{\prime}, x^{\prime}\right) d x^{\prime} d t^{\prime}, \\
S u(x) & =\sum_{k=0}^{d} \int_{0}^{T} \int_{\Omega} s_{k}\left(x, t^{\prime}, x^{\prime}\right) \partial_{t^{\prime}}^{k} u\left(t^{\prime}, x^{\prime}\right) d x^{\prime} d t^{\prime} \\
K c(t, x) & =\int_{\Omega} k\left(t, x, x^{\prime}\right) \cdot c\left(x^{\prime}\right) d x^{\prime} \\
Q c(x) & =\int_{\Omega} q\left(x, x^{\prime}\right) c\left(x^{\prime}\right) d x^{\prime}
\end{aligned}
$$

for suitable $g_{k} \in C^{\infty}([0, T) \times \Omega \times[0, T) \times \Omega), s_{k} \in C^{\infty}\left(\Omega \times[0, T) \times \Omega ; \mathbb{C}^{N_{+}}\right), k \in$ $C^{\infty}\left([0, T) \times \Omega \times \Omega ; \mathbb{C}^{N_{-}}\right)$, and $q \in C^{\infty}\left(\Omega \times \Omega ; \mathbb{C}^{N_{-}}, \mathbb{C}^{N_{+}}\right)$.

(b) The class $B^{m, d ; \lambda}\left((0, T) \times \Omega ; N_{-}, N_{+}\right)$consists of all operators

$$
\mathfrak{A}=\mathrm{Op}(\mathfrak{a})+\mathfrak{G}
$$

where $\mathfrak{a} \in R^{m, d ; \lambda}\left(\Omega \times \mathbb{R}^{n} ; N_{-}, N_{+}\right)$and $\mathfrak{G} \in B^{-\infty, d ; \lambda}\left((0, T) \times \Omega ; N_{-}, N_{+}\right)$.

(c) The class $B_{G}^{m, d ; \lambda}\left((0, T) \times \Omega ; N_{-}, N_{+}\right)$consists of all operators (5.1), where we additionally assume that $\mathfrak{a} \in R_{G}^{m, d ; \lambda}\left(\Omega \times \mathbb{R}^{n} ; N_{-}, N_{+}\right)$.

In case $N_{-}=N_{+}=0$, we also write $B^{m, d ; \lambda}((0, T) \times \Omega):=B^{m, d ; \lambda}((0, T) \times \Omega ; 0,0)$ and $B_{G}^{m, d ; \lambda}((0, T) \times \Omega):=B_{G}^{m, d ; \lambda}((0, T) \times \Omega ; 0,0)$, respectively.

REMARK 5.2. We have $B^{m, d ; \lambda}((0, T) \times \Omega) \subset L_{\mathrm{cl}}^{m}((0, T) \times \Omega)$ and $B_{G}^{m, d ; \lambda}((0, T) \times \Omega) \subset$ $L^{-\infty}((0, T) \times \Omega)$.

Elements $\mathfrak{A} \in B^{m, d ; \lambda}\left((0, T) \times \Omega ; N_{-}, N_{+}\right)$are called pseudodifferential boundary value problems of order $m$ and type $d$ (with degeneracy of type $\lambda$ ). Furthermore, elements $\mathfrak{A} \in B_{G}^{m, d ; \lambda}\left((0, T) \times \Omega ; N_{-}, N_{+}\right)$are called the regularizing ones (of the corresponding order and type, with degeneracy of type $\lambda$ ). 
Elements $\mathfrak{A} \in B^{m, d ; \lambda}\left((0, T) \times \Omega ; N_{-}, N_{+}\right)$are block matrices as indicated in (1.7).

Proposition 5.3. Each $\mathfrak{A} \in B^{m, d ; \lambda}\left((0, T) \times \Omega ; N_{-}, N_{+}\right)$extends by continuity to an operator

$$
\mathfrak{A}: \begin{gathered}
H_{\text {comp }}^{s, \delta ; \lambda}([0, T) \times \Omega) \\
H_{\text {comp }}^{s}\left(\Omega ; \mathbb{C}^{N_{-}}\right)
\end{gathered} \rightarrow \begin{array}{cc}
H_{\mathrm{loc}}^{s-m, \delta+m ; \lambda}([0, T) \times \Omega) \\
\rightarrow
\end{array} \quad \begin{gathered}
H_{\mathrm{loc}}^{s-m}\left(\Omega ; \mathbb{C}^{N_{+}}\right)
\end{gathered}
$$

for all $s>d-1 / 2$, cf. (1.8). If $\mathfrak{A} \in B^{m-1, d ; \lambda}\left((0, T) \times \Omega ; N_{-}, N_{+}\right)$, then the map (5.2) is compact.

Furthermore, if $\mathfrak{A} \in B^{m, d ; \lambda}\left((0, T) \times \Omega ; N_{-}, N_{+}\right)$is properly supported, then, throughout in (5.2), we are allowed to replace either "comp" with "loc" in the source space column or "loc" with "comp" in the target space column.

Again, asymptotic summation is possible:

Proposition 5.4. Let $\mathfrak{A}_{j} \in B^{m-j, d ; \lambda}\left((0, T) \times \Omega ; N_{-}, N_{+}\right)$for $j=0,1,2, \ldots$ Then there is an $\mathfrak{A} \in B^{m, d ; \lambda}\left((0, T) \times \Omega ; N_{-}, N_{+}\right)$such that, for all $J \in \mathbb{N}$,

$$
\mathfrak{A}-\sum_{j<J} \mathfrak{A}_{j} \in B^{m-J, d ; \lambda}\left((0, T) \times \Omega ; N_{-}, N_{+}\right) .
$$

Thereby, $\mathfrak{A}$ is uniquely determined modulo $B^{-\infty, d ; \lambda}\left((0, T) \times \Omega ; N_{-}, N_{+}\right)$. Moreover, if $\mathfrak{A}_{j} \in B_{G}^{m-j, d ; \lambda}\left((0, T) \times \Omega ; N_{-}, N_{+}\right)$for all $j$, then $\mathfrak{A} \in B_{G}^{m, d ; \lambda}\left((0, T) \times \Omega ; N_{-}, N_{+}\right)$.

We also write

$$
\mathfrak{A} \sim \sum_{j=0}^{\infty} \mathfrak{A}_{j} .
$$

Now, we are in a position to supply proofs of the main theorems, cf. Theorems 1.5 , 1.6, 1.8 .

5.1. Proof of Theorem 1.5. This is basically a consequence of Proposition 4.30 (c), since composition with elements from $B^{-\infty, d ; \lambda}\left((0, T) \times \Omega ; \tilde{N}, N_{+}\right)$from the left and with elements from $B^{-\infty, d^{\prime} ; \lambda}\left((0, T) \times \Omega ; N_{-}, \tilde{N}\right)$ from the right, respectively, is harmless. See also Schulze [20, Theorem 4.3.17].

5.2. Proof of Theorem 1.6. Each $\mathfrak{A} \in B^{m, d ; \lambda}\left((0, T) \times \Omega ; N_{-}, N_{+}\right)$possesses an interior principal symbol and a boundary principal symbol, respectively, defined by

$$
\begin{aligned}
\sigma_{\psi}^{m}(\mathfrak{A})(t, x, \tau, \xi) & :=\sigma_{\psi}^{m}(\mathfrak{a})(t, x, \tau, \xi), \\
\sigma_{b}^{m ; \lambda}(\mathfrak{A})(x, \xi) & :=\sigma_{b}^{m ; \lambda}(\mathfrak{a})(x, \xi),
\end{aligned}
$$

where $\mathfrak{A}$ is as in (5.1).

Definition 5.5. The principal symbol space $\Sigma B^{m, d ; \lambda}\left((0, T) \times \Omega ; N_{-}, N_{+}\right)$for the space $B^{m, d ; \lambda}\left((0, T) \times \Omega ; N_{-}, N_{+}\right)$consists of all couples $(a, \mathfrak{a})$, where $a \in S_{\mathrm{tr}}^{(m)}([0, T) \times$ $\left.\Omega \times\left(\mathbb{R}^{1+n} \backslash 0\right)\right)$ is such that

$$
a(t, x, \tau, \xi)=\tilde{a}\left(t, x, \tau, t^{l_{*}} \tau\right)
$$


for some $\tilde{a} \in S^{(m)}\left([0, T) \times \Omega \times\left(\mathbb{R}^{1+n} \backslash 0\right)\right)$ and $\mathfrak{a} \in C^{\infty}\left(\Omega \times\left(\mathbb{R}^{n} \backslash 0\right) ; B^{m, d ; \lambda}\left(\mathbb{R}_{+} ; N_{-}, N_{+}\right)\right)$ satisfies

$$
\mathfrak{a}(x, \nu \xi)=\nu^{m}\left(\begin{array}{cc}
\kappa_{\nu}^{(\delta+m)} & 0 \\
0 & 1_{\mathbb{C}^{N_{+}}}
\end{array}\right) \mathfrak{a}(x, \xi)\left(\begin{array}{cc}
\kappa_{\nu}^{(\delta)} & 0 \\
0 & 1_{\mathbb{C}^{N_{-}}}
\end{array}\right)^{-1}, \quad \nu>0,
$$

for any $\delta \in \mathbb{R}$. Moreover, it is required that

$$
\sigma_{\psi}^{m}(\mathfrak{a})(t, \tau ; x, \xi)=\tilde{a}\left(0, x, \tau, t^{l_{*}} \xi\right)
$$

With this definition, split exactness of the short sequence (1.10) is a consequence of the split exactness of the short sequence (4.31). See also Schulze [20, Proposition 4.3.14].

5.3. Proof of Theorem 1.8. Eventually, we provide the parametrix construction to the elliptic elements within the calculus.

Definition 5.6. A pseudodifferential boundary value problem $\mathfrak{A} \in B^{m, d ; \lambda}((0, T) \times \Omega$; $\left.N_{-}, N_{+}\right)$is called elliptic if $\sigma_{\psi}^{m}(\mathfrak{A})(t, x, \tau, \xi) \neq 0$ for $(t, x, \tau, \xi) \in(0, T) \times \Omega \times\left(\mathbb{R}^{1+n} \backslash 0\right)$ and, moreover, $\sigma_{b}^{m ; \lambda}(\mathfrak{A})(x, \xi)$ when considered as operator acting in (3.3) is invertible for all $(x, \xi) \in \Omega \times\left(\mathbb{R}^{n} \backslash 0\right)$.

Note that both conditions in this definition together imply that

$$
\tilde{\sigma}_{\psi}^{m ; \lambda}(\mathfrak{A})(t, x, \tau, \xi) \neq 0 \quad \text { for }(t, x, \tau, \xi) \in[0, T) \times \Omega \times\left(\mathbb{R}^{1+n} \backslash 0\right)
$$

(i.e., invertibility holds up to $t=0$ ) thanks to the compatibility condition (5.4).

Lemma 5.7. Let $\mathfrak{A} \in B^{m, d ; \lambda}\left((0, T) \times \Omega ; N_{-}, N_{+}\right)$be elliptic. Then

$$
\left(\sigma_{\psi}^{m}(\mathfrak{A})^{-1}, \sigma_{b}^{m ; \lambda}(\mathfrak{A})^{-1}\right) \in \Sigma B^{-m,(d-m)^{+} ; \lambda}\left((0, T) \times \Omega ; N_{+}, N_{-}\right),
$$

with the inverses taken pointwise.

Now, assume that $\mathfrak{A} \in B^{m, d ; \lambda}\left((0, T) \times \Omega ; N_{-}, N_{+}\right)$is elliptic in the sense of Definition 5.6. Then, according to Theorem 1.6 and the previous lemma, we can choose a $\mathfrak{B}_{0} \in B^{-m,(d-m)^{+} ; \lambda}\left((0, T) \times \Omega ; N_{+}, N_{-}\right)$such that

$$
\sigma_{\psi}^{-m}\left(\mathfrak{B}_{0}\right)=\sigma_{\psi}^{m}(\mathfrak{A})^{-1}, \quad \sigma_{b}^{-m ; \lambda}\left(\mathfrak{B}_{0}\right)=\sigma_{b}^{m ; \lambda}(\mathfrak{A})^{-1} .
$$

Then, by virtue of Theorems 1.5, 1.6,

$$
\mathfrak{N}_{0}:=1-\mathfrak{B}_{0} \mathfrak{A} \in B^{-1, \max \{m, d\} ; \lambda}\left((0, T) \times \Omega ; N_{-}, N_{-}\right) .
$$

We get $\mathfrak{N}_{0}^{j} \in B^{-j, \max \{m, d\} ; \lambda}\left((0, T) \times \Omega ; N_{-}, N_{-}\right)$for all $j \in \mathbb{N}, j \geq 1$, so that we can further choose an $\mathfrak{N} \in B^{-1, \max \{m, d\} ; \lambda}\left((0, T) \times \Omega ; N_{-}, N_{-}\right)$satisfying

$$
\mathfrak{N} \sim \sum_{j=1}^{\infty} \mathfrak{N}_{0}^{j}
$$

in view of Proposition 5.4. Then

$$
\mathfrak{B}:=(1+\mathfrak{N}) \mathfrak{B}_{0} \in B^{-m,(d-m)^{+} ; \lambda}\left((0, T) \times \Omega ; N_{+}, N_{-}\right)
$$

is a left parametrix to $\mathfrak{A}$, i.e., we have

$$
\mathfrak{B A}-1 \in B^{-\infty, \max \{m, d\} ; \lambda}\left((0, T) \times \Omega ; N_{-}, N_{-}\right) .
$$


Starting with $\mathfrak{N}_{0}^{\prime}:=1-\mathfrak{A B}_{0} \in B^{-1,(d-m)^{+} ; \lambda}\left((0, T) \times \Omega ; N_{+}, N_{+}\right)$, in a similar way a right parametrix $\mathfrak{B}^{\prime} \in B^{-m,(d-m)^{+} ; \lambda}\left((0, T) \times \Omega ; N_{+}, N_{-}\right)$to $\mathfrak{A}$, i.e., we have

$$
\mathfrak{A} \mathfrak{B}^{\prime}-1 \in B^{-\infty,(d-m)^{+} ; \lambda}\left((0, T) \times \Omega ; N_{+}, N_{+}\right),
$$

is constructed. We then obtain

$$
\mathfrak{B}-\mathfrak{B}^{\prime}=(\mathfrak{B A}-1) \mathfrak{B}^{\prime}-\mathfrak{B}\left(\mathfrak{A} \mathfrak{B}^{\prime}-1\right) \in B^{-\infty,(d-m)^{+} ; \lambda}\left((0, T) \times \Omega ; N_{+}, N_{-}\right) .
$$

This finishes the proof of Theorem 1.8.

We conclude with the following example:

EXAMPLE 5.8. Let

$$
A=\partial_{t}^{2}+2 \sum_{j=1}^{n} c_{j}(t, x) t^{l_{*}} \partial_{t} \partial_{x_{j}}+\sum_{i, j=1}^{n} a_{i j}(t, x) t^{2 l_{*}} \partial_{x_{i}} \partial_{x_{j}}+\sum_{j=1}^{n} b_{j}(t, x) t^{l_{*}-1} \partial_{x_{j}},
$$

where $a_{i j}, c_{j}, b_{j} \in C^{\infty}([0, T) \times \Omega)$ are real-valued and

$$
\sum_{i, j}^{n} a_{i j}(t, x) \xi_{i} \xi_{j}>\left(\sum_{j=1}^{n} c_{j}(t, x) \xi_{j}\right)^{2}, \quad(t, x, \xi) \in[0, T) \times \Omega \times \mathbb{R}^{n},|\xi|=1 .
$$

Then $A \in B^{2,0 ; \lambda}((0, T) \times \Omega)$ and, by virtue of Example 3.10 ,

$$
\left(\begin{array}{c}
A \\
R \gamma_{0}
\end{array}\right): C^{\infty}([0, T) \times \Omega) \rightarrow \begin{gathered}
C^{\infty}([0, T) \times \\
C^{\infty}(\Omega)
\end{gathered}
$$

is an elliptic pseudodifferential boundary value problem in $B^{2,1 ; \lambda}((0, T) \times \Omega ; 0,1)$ (here, $R \in L_{\mathrm{cl}}^{3 / 2}(\Omega)$ is properly supported and invertible as operator from $H_{\mathrm{loc}}^{3 / 2}(\Omega)$ to $\left.H_{\mathrm{loc}}^{0}(\Omega)\right)$ if and only if

$$
b(x, \xi) \notin D_{a(x, \xi), c(x, \xi)}, \quad(x, \xi) \in \Omega \times \mathbb{R}^{n},|\xi|=1,
$$

where

$$
a(x, \xi):=\sum_{i, j=1}^{n} a_{i j}(0, x) \xi_{i} \xi_{j}, \quad b(x, \xi):=\sum_{j=1}^{n} b_{j}(0, x) \xi_{j}, \quad c(x, \xi):=\sum_{j=1}^{n} c_{j}(0, x) \xi_{j} .
$$

In case $(5.5),(5.7)$ are fulfilled, the pseudodifferential boundary value problem (5.6) admits a parametrix belonging to $B^{-2,0 ; \lambda}((0, T) \times \Omega ; 1,0)$.

\section{References}

[1] R. BeAls, A general calculus of pseudodifferential operators, Duke Math. J. 42 (1975), 1-42.

[2] L. Boutet DE Monvel, Boundary problems for pseudo-differential operators, Acta Math. 126 (1971), 11-51.

[3] L. Boutet De Monvel, Hypoelliptic operators with double characteristics and related pseudodifferential operators, Comm. Pure Appl. Math. 27 (1974), 585-639.

[4] M. Dreher, An application of the theory of edge Sobolev spaces to weakly hyperbolic operators, this volume, 105-119.

[5] M. Dreher and I. WiTt, Edge Sobolev spaces and weakly hyperbolic equations, Ann. Mat. Pura Appl. (4) 180 (2002), 451-482. 
[6] V. GRushin, On a class of elliptic pseudodifferential operators degenerate on a submanifold (in Russian), Mat. Sb. (N.S.) 84 (1971), 163-195; English transl.: Math. USSR-Sb. 13 (1971), $155-185$.

[7] V. Grushin, Hypoelliptic differential equations and pseudodifferential operators with operator-valued symbols (in Russian), Mat. Sb. (N.S.) 88 (1972), 504-521; English transl.: Math. USSR-Sb. 17 (1972), 497-514.

[8] N. Hanges, Propagation of singularities for a class of operators with double characteristics, In: Seminar on Singularities of Linear Partial Differential Equations, L. Hörmander (ed.), Ann. of Math. Studies 91, Princeton Univ. Press, Princeton, 1979, 113-126.

[9] T. Hirschmann, Pseudo-differential operators and asymptotics on manifolds with corners V a. Supplement: Pseudo-differential operator calculus with exit conditions, Report R-Math 04/91, Karl-Weierstraß-Institut für Mathematik, Berlin, 1991.

[10] L. Hörmander, The Weyl calculus of pseudodifferential operators, Comm. Pure Appl. Math. 32 (1979), 360-444.

[11] L. Hörmander, The Analysis of Linear Differential Operators III, Grundlehren Math. Wiss. 274, Springer, Berlin, 1985.

[12] V. Ya. IvrII, V. M. Petkov, Necessary conditions for the correctness of the Cauchy problem for non-strictly hyperbolic equations (in Russian), Uspekhi Mat. Nauk 29 (1974), no. 5, 3-70; English transl.: Russian Math. Surveys 29 (1974), no. 5, 1-70.

[13] M. Joshi, A symbolic construction of the forward fundamental solution of the wave operator, Comm. Partial Differential Equations 23 (1998), 1349-1417.

[14] H. Kumano-go, Pseudo-differential Operators, MIT Press, Cambridge, Mass., 1981.

[15] S. Levendorskit, Degenerate Elliptic Equations, Math. Appl. 258, Kluwer, Dordrecht, 1993.

[16] M. Mascarello and L. Rodino, Partial Differential Equations with Multiple Characteristics, Math. Top. 13, Akademie Verlag, Berlin, 1997.

[17] F. Olver, Asymptotics and Special Functions, Academic Press, New York, 1974.

[18] S. Rempel and B.-W. Schulze, Index Theory of Elliptic Boundary Problems, AkademieVerlag, Berlin, 1982.

[19] E. Schrohe and B.-W. Schulze, Boundary value problems in Boutet de Monvel's algebra for manifolds with conical singularities I, In: Pseudo-Differential Calculus and Mathematical Physics, M. Demuth, E. Schrohe, and B.-W. Schulze (eds.), Math. Top. 5, Akademie Verlag, Berlin, 1994, 97-209.

[20] B.-W. Schulze, Boundary Value Problems and Singular Pseudo-differential Operators, Pure Appl. Math., J. Wiley, Chichester, 1998.

[21] J. Sjöstrand, Parametrices for pseudodifferential operators with multiple characteristics, Ark. Mat. 12 (1974), 85-130.

[22] M. TAYlor, Pseudodifferential Operators, Princeton Math. Ser. 34, Princeton Univ. Press, Princeton, 1981.

[23] M. VishiK and V. Grushin, On a class of higher order degenerate elliptic equations, Mat. Sb. 79 (1969), 3-36 (in Russian); English transl.: Math. USSR-Sb. 8 (1969), 1-32.

[24] K. Yagdjian, The Cauchy Problem for Hyperbolic Operators. Multiple Characteristics. Micro-local Approach, Math. Top. 12, Akademie Verlag, Berlin, 1997. 